Pacific Journal of Mathematics

SPECTRAL CONCENTRATION FOR SELF-ADJOINT 


\title{
SPECTRAL CONCENTRATION FOR SELF-ADJOINT OPERATORS
}

\author{
R. C. RIDDELL
}

If the resolvent of a (not necessarily bounded) self-adjoint operator $H_{\kappa}$ converges strongly to the resolvent of a selfadjoint operator $H$, and if $\lambda$ is an isolated eigenvalue of $H$ of multiplicity $m<\infty$, then although $H_{\kappa}$ need not have an eigenvalue near $\lambda$, the spectrum of $H_{\kappa}$ will in some cases become "concentrated" near $\lambda$ as $\kappa$ is reduced. In fact, there exist sets $C_{\kappa}$ with Lebesgue measure $o\left(\kappa^{p}\right), p \geqq 0$, such that the spectral projection assigned by $H_{\kappa}$ to $C_{\kappa}$ converges strongly as $\kappa \rightarrow 0$ to the projection on the $\lambda$-eigenspace of $H$, if and only if there exist $m$ pairs $\left(\lambda_{j \kappa}, \varphi_{j \kappa}\right), j=1, \cdots, m$, where $\lambda_{j \kappa} \rightarrow \lambda$, the $\varphi_{j k}$ are nearly-orthogonal unit vectors converging strongly to the $\lambda$-eigenspace, and $\left\|\left(H_{\kappa}-\lambda_{j \kappa}\right) \varphi_{j \kappa}\right\|=o\left(\kappa^{p}\right)$. In this case, $C_{\kappa}$ may be taken as the union of intervals about the $\lambda_{j \kappa}$, and the $\lambda_{j k}$ are essentially the only numbers associated in this way with "pseudoeigenvectors" $\varphi_{j k}$ of $H_{k}$. The result is applied to the weak-quantization problem in the theory of the Stark effect, where $H$ is the Hamiltonian operator for the hydrogen atom, and $H_{\kappa}$ is the same for the atom in a uniform electric field which vanishes with $\kappa$.

In $\S 1$ the basic notions of spectral concentration and pseudoeigenvectors are discussed, and some simple lemmas are proved relating the two. The theorem quoted above is proved in $\S 2$ (Theorem 2.7), and the question arises how the pairs $\left(\lambda_{j \kappa}, \varphi_{j \kappa}\right)$ can be constructed. For a family of the form $H_{\kappa}=H+\kappa V$, this construction is carried out in $\S 3$ by means of the formal perturbation process applied to the unperturbed eigenvalue $\lambda$. In $\S 4$, the special case is considered in which $\lambda$ is stable, i.e. $H_{\kappa}$ has $m$ eigenvalues in a neighborhood of $\lambda$; asymptotic estimates of these perturbed eigenvalues follow easily. Finally, in $\S 5$, the theory is applied to the generic example, the family of operators appearing in the Stark effect. Here the spectrum of $H_{k}$ is purely continuous and covers the real line, and so technically the perturbed system has no stationary states. Yet the lines in the physical spectrum of hydrogen persist when a weak electric field is applied, each one splitting into several quite sharp lines. These lines can be traced to the existence of "almost stationary" states, which are represented by the pseudoeigenvectors $\varphi_{j \kappa}$ mentioned above. It is stressed that there are many other vectors $\psi_{k}$ which represent "almost stationary" states, in the sense that the solution of the equation of motion with initial state $\psi_{\kappa}$ remains close to $\psi_{k}$ for a long time. It is the fact that no sequence $\varphi_{j \kappa_{n}}$ converges weakly to zero $\left(\kappa_{n} \rightarrow 0\right)$ 
which distinguishes the $\varphi_{j \kappa}$.

Perturbation problems of this sort, involving continuous spectra, were considered in [3] and [4]. In [6], Kato proved for the operators here considered in $\S 5$ that the formal solutions of the perturbation equations are essentially the only almost stationary states, although in a somewhat different sense of "essentially" from ours. The notion of spectral concentration originated with Titchmarsh [10], who proved that the spectrum of the operator for hydrogen in an electric field is concentrated on intervals of length $o\left(\kappa^{p}\right), p<2$, centered on the first order solutions $\lambda_{j \kappa}$ of the perturbation equations. Conley and Rejto proved an abstract version of Titchmarsh's result in [1].

Having completed a preliminary version of this article, the author received from Professors Conley and Rejto a preprint [2] containing similar results with different proofs. One such result was the "if" part of Theorem 2.7; the "only if" part was asserted for the case $m=1$ and posed as a problem for general $m$. Their condition that the pseudoeigenvectors converge strongly to the unperturbed eigenspace, less restrictive than the one originally used by the author, facilitated the proof of the theorem in the reverse direction.

The author would like to extend cordial thanks to Professor T. Kato for valuable suggestions and encouragement through the course of the work.

1. Spectral concentration and pseudoeigenvectors. $H$ will denote a self-adjoint operator, in general unbounded, with domain $\mathscr{D}(H)$ in a Hilbert space $\mathscr{H}$. $J$ will always denote a Borel set of real numbers, and the spectrum of $H$ in $J$, that is, the intersection of $J$ with the spectrum of $H$, will be denote by $\Sigma(J) . E$ will denote the spectral family of $H$, assumed continuous from the right: $E(\mu)=$ $E(\mu+)$. The same symbol will be used for the measure induced by $E$, with $E[J]$ denoting the projection assigned by this measure to $J$. When $J$ is the closed interval $\lambda-\delta \leqq \mu \leqq \lambda+\delta$, we shall write $E[\lambda, \delta]$ for $E[J]=E(\lambda+\delta)-E((\lambda-\delta)-)$. In case $\delta=0, E[\lambda] \equiv$ $E[\lambda, 0]$ is zero unless $\lambda$ is an eigenvalue of $H$, in which case $E[\lambda]$ is the projection on the $\lambda$-eigenspace.

Analogous symbols with a suffix $\kappa$ will be used for a family of self-adjoint operators $\left\{H_{\kappa}: 0<\kappa \leqq \kappa_{0}\right\}$. For instance, $\Sigma_{\kappa}(J)$ will denote the spectrum of $H_{\kappa}$ in $J$.

Convergence of vectors will always be strong convergence, unless marked $\stackrel{w}{\longrightarrow}$ for weak convergence. Landau symbols $o\left(\kappa^{p}\right)$ for vectors will be used in the sense of strong convergence. The symbol $\stackrel{s}{\longrightarrow}$ will denote strong convergence of bounded operators. Limits of functions of $\kappa$ will always be taken as $\kappa \rightarrow 0+$. 
Our basic notions are made precise in the following:

DEFINITION 1.1. Let $\left\{H_{k}\right\}$ be a family of self-adjoint operators, $o<\kappa \leqq \kappa_{0}$, and let $\left\{C_{\kappa}\right\}$ be a family of Borel subsets of $J$. We say $\Sigma_{\kappa}(J)$ is concentrated on $C_{\kappa}$ provided $E_{\kappa}\left[J-C_{\kappa}\right] \stackrel{s}{\longrightarrow} 0$.

DEFINITION 1.2. Let $\left\{\varphi_{k}\right\}$ be a family of unit vectors such that $\varphi_{\kappa} \in \mathscr{D}\left(H_{\kappa}\right)$ and let $\left\{\lambda_{\kappa}\right\}$ be a family of real numbers. If, for a real number $p \geqq 0,\left(H_{\kappa}-\lambda_{\kappa}\right) \varphi_{\kappa}=o\left(\kappa^{p}\right)$, then $\left\{\varphi_{\kappa}\right\}$ is a pseudoeigenvector of $\left\{H_{\kappa}\right\}$ of order $p$ and $\left\{\lambda_{k}\right\}$ a corresponding pseudoeigenvalue. For short we shall say simply that $\left\{\left(\lambda_{\kappa}, \varphi_{\kappa}\right)\right\}$ is a $p$-pair for $\left\{H_{\kappa}\right\}$.

It is essential to realize that each of these definitions prescribes the behavior of a certain function of $\kappa$ in the limit as $\kappa \rightarrow 0$, and that the quantities could be changed more or less at random for a given $\kappa>0$ without affecting this behavior. Thus it does not make sense to say that $\varphi_{k}$ is a pseudoeigenvector of $H_{k}$. Nevertheless we shall do so, but only to avoid writing in the braces each time; the reader will easily see where they belong.

Of course a $p$-pair for $H_{\kappa}$ is also a $q$-pair for $q \leqq p$, and an eigenvalue-eigenvector pair constitutes a $p$-pair for any $p$. The basic connection between $p$-pairs and spectral concentration is made apparent in the following elementary result, which asserts that a high-order pseudoeigenvector is almost in the range of the spectral projector supported by a small interval about its corresponding pseudoeigenvalue.

Lemma 1.3. Let $\left(\lambda_{\kappa}, \varphi_{\kappa}\right)$ be a p-pair for $H_{\kappa}$, and let $\eta_{\kappa}$ be an arbitrary family of nonnegative real numbers. Then

$$
\eta_{\kappa}\left(1-E_{\kappa}\left[\lambda_{\kappa}, \eta_{\kappa}\right]\right) \varphi_{\kappa}=o\left(\kappa^{p}\right)
$$

Proof. Let $K_{\kappa}$ denote the complement of the interval

$$
\lambda_{\kappa}-\eta_{\kappa}<\mu<\lambda_{\kappa}+\eta_{\kappa}
$$

Then

$$
\begin{aligned}
o\left(\kappa^{2 p}\right) & =\left\|\left(H_{\kappa}-\lambda_{\kappa}\right) \varphi_{\kappa}\right\|^{2} \\
& =\int_{-\infty}^{\infty}\left(\mu-\lambda_{\kappa}\right)^{2} d\left\|E_{\kappa}(\mu) \varphi_{\kappa} !\right\|^{2} \\
& \geqq \int_{K_{\kappa}}\left(\mu-\lambda_{\kappa}\right)^{2} d\left\|E_{\kappa}(\mu) \varphi_{\kappa}\right\|^{2} \\
& \geqq \eta_{\kappa}^{2} \int_{K_{\kappa}} d\left\|E_{\kappa}(\mu) \varphi_{\kappa}\right\|^{2} \\
& \geqq \eta_{\kappa}^{2}\left\|\left(1-E_{\kappa}\left[\lambda_{\kappa}, \eta_{\kappa}\right]\right) \varphi_{\kappa}\right\|^{2} .
\end{aligned}
$$


It is a familiar fact that if $(H-\lambda) \varphi$ is small in norm, then there must be a point of the spectrum of $H$ near $\lambda$. The following result can be regarded as a generalization of this to the case of a family of operators.

LEMmA 1.4. Let $J$ be an open set of real numbers, and suppose that $\Sigma_{\kappa}(J)$ is concentrated on $C_{\kappa}$. Let $\left(\lambda_{\kappa}, \varphi_{\kappa}\right)$ be a p-pair for $H_{\kappa}$ such that $\lambda_{\kappa}$ is eventually in a closed subset of $J$ and such that there is no sequence $\kappa_{n} \rightarrow 0$ for which $\varphi_{\kappa_{n}} \stackrel{w}{\longrightarrow} 0$. Then $\lambda_{\kappa}$ is within a distance $o\left(\kappa^{p}\right)$ of $C_{\kappa}$.

Proof. Put $\eta_{\kappa}=1 / 2$ dist $\left(\lambda_{\kappa}, C_{\kappa}\right)$. For those $\kappa$ for which $\eta_{\kappa}=0$ there is nothing to prove. If $\eta_{\kappa} \neq 0$ then the interval $\lambda_{\kappa}-\eta_{\kappa} \leqq \mu \leqq$ $\lambda_{\kappa}+\eta_{\kappa}$ is disjoint from $C_{\kappa}$, and so $E_{\kappa}\left[\lambda_{\kappa}, \eta_{\kappa}\right]+E_{\kappa}\left[C_{\kappa}\right] \leqq 1$, the identity operator. From Lemma 1.3,

$$
o\left(\kappa^{2 p}\right)=\eta_{\kappa}^{2}\left\|\left(1-E_{\kappa}\left[\lambda_{\kappa}, \eta_{\kappa}\right]\right) \varphi_{\kappa}\right\|^{2} \geqq \eta_{\kappa}^{2}\left\|E_{\kappa}\left[C_{\kappa}\right] \varphi_{\kappa}\right\|^{2},
$$

and it would follow that $\eta_{\kappa}=o\left(\kappa^{p}\right)$ if $\left\|E_{\kappa}\left[C_{\kappa}\right] \varphi_{\kappa}\right\| \geqq \varepsilon>0$.

To see this, we first use the hypothesis on $\lambda_{\kappa}$ to find a number $\rho>0$ such that $\lambda_{\kappa}-\rho \leqq \mu \leqq \lambda_{\kappa}+\rho$ is contained in $J$, for $\kappa$ small enough. Then by Lemma 1.3,

$$
\left\|\left(1-E_{\kappa}[J]\right) \varphi_{\kappa}\right\| \leqq\left\|\left(1-E_{\kappa}\left[\lambda_{\kappa}, \rho\right]\right) \varphi_{\kappa}\right\|=\frac{1}{\rho} o(1) .
$$

Now suppose that there is no $\varepsilon>0$ for which $\left\|E_{\kappa}\left[C_{\kappa}\right] \varphi_{\kappa}\right\|>\varepsilon$. Then there is a sequence $\kappa_{n} \rightarrow 0$ such that $\left\|E_{\kappa_{n}}\left[C_{\kappa_{n}}\right] \varphi_{\kappa_{n}}\right\| \rightarrow 0$. Using this and the convergence just proved, we obtain

$$
\begin{aligned}
\varphi_{\kappa_{n}} & =E_{\kappa_{n}}[J] \varphi_{\kappa_{n}}+o(1) \\
& =E_{\kappa_{n}}\left[J-C_{\kappa_{n}}\right] \varphi_{\kappa_{n}}+o(1)+o(1) .
\end{aligned}
$$

Let $\varphi$ be any unit vector in $\mathscr{H}$. Then

$$
\begin{aligned}
\left|\left(\varphi, \varphi_{\kappa_{n}}\right)\right| & =\left|\left(\varphi, E_{\kappa_{n}}\left[J-C_{\kappa_{n}}\right] \varphi_{\kappa_{n}}\right)\right|+o(1) \\
& =\left|\left(E_{\kappa_{n}}\left[J-C_{\kappa_{n}}\right] \varphi, \varphi_{\kappa_{n}}\right)\right|+o(1) \\
& \leqq\left\|E_{\kappa_{n}}\left[J-C_{\kappa_{n}}\right] \varphi\right\|+o(1) .
\end{aligned}
$$

But $E_{\kappa_{n}}\left[J-C_{\kappa_{n}}\right] \stackrel{s}{\longrightarrow} 0$, hence $\varphi_{\kappa_{n}} \stackrel{w}{\longrightarrow} 0$, a contradiction. Thus $\left\|E_{\kappa}\left[C_{\kappa}\right] \varphi_{\kappa}\right\|$ must be bounded away from 0 , and the lemma is proved.

The hypothesis on $\varphi_{\kappa}$ cannot be done away with. For example, if $\mathscr{H}=L_{2}(0,1)$ and $H_{\kappa}$ is multiplication by the characteristic function $\chi_{\kappa}$ of the interval $(0, \kappa)$, then $E_{\kappa} \stackrel{s}{\longrightarrow} 0$ and it is easy to check that the whole spectrum of $H_{\kappa}$ is concentrated on $C_{\kappa} \equiv\{0\}$. On the other 
hand, $H_{\kappa}$ even has an eigenvalue $\lambda_{\kappa} \equiv 1$, with unit eigenvector, e.g., $\varphi_{\kappa}=\kappa^{-1 / 2} \chi_{\kappa}$; and this $\lambda_{\kappa}$ is nowhere near $C_{\kappa}$. Of course $\varphi_{\kappa} \stackrel{w}{\longrightarrow} 0$.

It should be noted that no assumptions have yet been made concerning the convergence of the operators $H_{\kappa}$ as $\kappa \rightarrow 0$.

2. Concentration at an isolated eigenvalue. We shall now consider a family $H_{\kappa}$ for which the resolvents $R_{\kappa}(\zeta)=\left(H_{\kappa}-\zeta\right)^{-1}$ converge strongly to the resolvent $R(\zeta)=(H-\zeta)^{-1}$ of a self-adjoint operator $H$, for each complex number $\zeta$ which is at a positive distance from the union of the spectra of $H$ and $H_{\kappa}$. This convergence holds for all such $\zeta$ if it holds for any, and it does hold if $H_{\kappa}$ and $H$ are bounded and $H_{\kappa} \stackrel{s}{\longrightarrow} H$ (see [7], p. 429). Thus it is a reasonable generalization of strong convergence to the case of unbounded operators. It will be denoted by $H_{\kappa} \stackrel{r}{\longrightarrow} H$. We quote without proof the following sufficient conditions ([7], p. 429, p. 453):

LEMMA 2.1. Suppose that there is a dense linear set

$$
\mathscr{D} \subset \mathscr{D}(H) \cap \mathscr{D}\left(H_{\kappa}\right)
$$

for sufficiently small $\kappa$, such that $H_{\kappa} \varphi \rightarrow H \varphi$ for each $\varphi \in \mathscr{D}$. Then $H_{\kappa} \stackrel{r}{\longrightarrow} H$ provided that either of the following holds:

(a) the restriction of $H$ to $\mathscr{D}$ is essentially self-adjoint, or

(b) $H_{\kappa} \geqq H \geqq$ constant, and the restriction of $H$ to $\mathscr{D}$ has $H$ as its Friedrichs extension.

Condition (a) means that the closure of $H \mid \mathscr{D}$ is self-adjoint (and hence equal to $H$ ), and it requires that $H \mathscr{D}$ be dense in $\mathscr{H}$. Condition (b) is stronger in that it requires the operators to be semibounded, but weaker in that only $(H+\text { const. })^{1 / 2} \mathscr{D}$ need be dense in $\mathscr{C}$.

Our aim is to study the spectrum of $H_{\kappa}$ in the neighborhood of an eigenvalue of $H$. First we notice that the hypothesis $H_{\kappa} \stackrel{r}{\longrightarrow} H$ guarantees a rather close connection between eigenvalues of $H$ and certain pseudoeigenvalues of $H_{\kappa}$.

Lemma 2.2. Suppose that $H_{\kappa} \stackrel{r}{\longrightarrow} H$. If $\left(\lambda_{\kappa}, \varphi_{\kappa}\right)$ is a 0-pair for $H_{\kappa}$ such that $\varphi_{\kappa}$ does not converge weakly to 0 , then $H$ has an eigenvalue $\lambda$ with associated eigenvector $\varphi$ such that $\lambda_{\kappa_{n}} \rightarrow \lambda$ and $\varphi_{\kappa_{n}} \stackrel{w}{\longrightarrow} \varphi$ for some sequence $\kappa_{n} \rightarrow 0$. Conversely, if $\lambda$ and $\varphi$ are an eigenvalue and an associated unit eigenvector of $H$, then $H_{\kappa}$ has such a 0-pair $\left(\lambda_{\kappa}, \varphi_{\kappa}\right)$; in fact $\lambda_{\kappa}$ and $\varphi_{\kappa}$ can be chosen so that $\lambda_{\kappa} \equiv \lambda, \varphi_{\kappa} \rightarrow \varphi$.

Proof. If $\left\|\left(H_{\kappa}-\lambda_{\kappa}\right) \varphi_{\kappa}\right\|=f(\kappa)$ where $f(\kappa) \rightarrow 0$, then $g(\kappa)$ can be 
chosen so that $g(\kappa) \rightarrow 0$ and $f(\kappa) / g(\kappa) \rightarrow 0$. Lemma 1.3 with $\eta_{\kappa}=g(\kappa)$ then gives $\|\left(1-E_{\kappa}\left[\lambda_{\kappa}, o(1)\right] \varphi_{\kappa} \|=o(1)\right.$. From this and the spectral formula for $H_{\kappa}$, it follows easily that $\left(R_{\kappa}(i)-\rho_{\kappa}\right) \varphi_{\kappa}=o(1)$, where $\rho_{\kappa}=\left(\lambda_{\kappa}-i\right)^{-1}$. In particular, for any sequence $\kappa_{n} \rightarrow 0$,

$$
R_{\kappa_{n}}(i) \varphi_{\kappa_{n}}-\rho_{\kappa_{n}} \varphi_{\kappa_{n}} \stackrel{w}{\longrightarrow} 0 \text {. }
$$

Next, by hypothesis there is some open weak neighborhood $N$ of $0 \in \mathscr{H}$ and some sequence $\kappa_{n} \rightarrow 0$ such that $\varphi_{\kappa_{n}} \notin N$. Since $\left\|\varphi_{\kappa_{n}}\right\|=1$, there is a subsequence, again denoted by $\varphi_{\kappa_{n}}$, which converges weakly; the weak limit $\varphi$ is not zero. The numbers $\rho_{\kappa_{n}}$ are all bounded in absolute value by 1 , and so $\rho_{\kappa_{n}}\left(\varphi_{\kappa_{n}}-\varphi\right) \stackrel{w}{\longrightarrow} 0$.

Since $R_{\kappa_{n}}(i)^{*}=R_{\kappa_{n}}(-i) \stackrel{s}{\longrightarrow} R(-i)$ and $\psi_{n}=\varphi-\varphi_{\kappa_{n}} \stackrel{w}{\longrightarrow} 0$, we have for each $\psi \in \mathscr{H}$

$$
\begin{aligned}
\left(R_{\kappa_{n}}(i) \psi_{n}, \psi\right) & =\left(\psi_{n}, R_{\kappa_{n}}(-i) \psi\right) \\
& =\left(\psi_{n},\left[R_{\kappa_{n}}(-i)-R(-i)\right] \psi\right)+\left(\psi_{n}, R(-i) \psi\right) \longrightarrow 0
\end{aligned}
$$

that is, $R_{\kappa_{n}}(i) \varphi-R_{\kappa_{n}}(i) \varphi_{\kappa_{n}} \stackrel{w}{\longrightarrow} 0$.

Finally, $H_{\kappa} \stackrel{r}{\longrightarrow} H$ gives, in particular, that $R(i) \varphi-R_{\kappa_{n}}(i) \varphi \stackrel{w}{\longrightarrow} 0$.

Adding the four sequences which have been shown to converge weakly to 0 , we obtain $\rho_{\kappa_{n}} \varphi \stackrel{w}{\longrightarrow} R(i) \varphi$. With $\psi=\|\varphi\|^{-1} \varphi$, this gives $\rho_{\kappa_{n}}=\left(\rho_{\kappa_{n}} \psi, \psi\right) \rightarrow(R(i) \psi, \psi)$. Then $(R(i) \psi, \psi) \neq 0$, for otherwise $(H-i)^{-1} \psi=$ weak limit $\rho_{\kappa_{n}} \psi=0$, and $\psi=0$, impossible.

Set $\lambda=i+(R(i) \psi, \psi)^{-1}$. Then $\left(\lambda_{\kappa_{n}}-i\right)^{-1}=\rho_{\kappa_{n}} \rightarrow(R(i) \psi, \psi)=$ $(\lambda-i)^{-1}$, and so $\lambda_{\kappa_{n}} \rightarrow \lambda$. Moreover, $(H-i)^{-1} \psi=$ weak limit $\rho_{\kappa_{n}} \psi=$ $(\lambda-i)^{-1} \psi$, and so $H \varphi=\lambda \varphi$, as required.

To prove the converse, let $\lambda$ be an eigenvalue of $H$ with an associated unit eigenvector $\varphi$. Set $\psi_{\kappa}=R_{k}(i)(H-i) \varphi$. Then $H_{\kappa} \stackrel{r}{\longrightarrow} H$ gives $\psi_{\kappa} \rightarrow \varphi$; moreover $\psi_{\kappa} \in \mathscr{D}\left(H_{\kappa}\right)$ and

$$
\begin{aligned}
\left(H_{\kappa}-\lambda\right) \psi_{\kappa} & =\left(H_{\kappa}-i\right) \psi_{\kappa}-(\lambda-i) \psi_{\kappa} \\
& =(H-i) \varphi-(\lambda-i) \varphi+(\lambda-i)\left(\varphi-\psi_{\kappa}\right) \\
& =(\lambda-i)\left(\varphi-\psi_{\kappa}\right) \\
& =o(1)
\end{aligned}
$$

since $H \varphi=\lambda \varphi$. Thus $\left(\lambda_{\kappa} \equiv \lambda, \varphi_{\kappa}=\left\|\psi_{\kappa}\right\|^{-1} \psi_{\kappa}\right)$ is the 0-pair for $H_{\kappa}$ whose existence was asserted.

Turning now to concentration of the spectrum of $H_{\kappa}$, we note that $H_{\kappa} \stackrel{r}{\longrightarrow} H$ implies $E_{\kappa}(\mu) \stackrel{s}{\longrightarrow} E(\mu)$ provided that $\mu$ is not an eigenvalue of $H$ (see [7], pp. 432-433; with the hypothesis (a) of Lemma 2.1, this is a classical result due to Rellich). If $J=[\alpha, \beta]$ is an interval containing an eigenvalue $\lambda$ of $H$ in its interior but other- 
wise disjoint from the spectrum of $H$ (an isolating interval for $\lambda$ ), then this convergence gives $E_{\kappa}(\alpha) \stackrel{s}{\longrightarrow} E(\alpha), E(\beta) \stackrel{s}{\longrightarrow} E_{\kappa}(\beta)$ and so $E_{\kappa}[J] \stackrel{s}{\longrightarrow} E[J]=E[\lambda]$, the eigenprojection associated with $\lambda$. Of course the same is true if $J$ is replaced by any smaller isolating interval $J^{\prime}$; thus $\Sigma_{\kappa}(J)$ is concentrated on any such $J^{\prime} \subset J$. Our concern is with the possibility that $\Sigma_{\kappa}(J)$ is in fact concentrated on sets $C_{\kappa}$ whose Lebesgue measure $\nu\left(C_{\kappa}\right)$ tends to zero with some definite order of continuity in $\kappa$. To settle this we shall have to assume that $\lambda$ has finite multiplicity.

ASSUMPTION 2.3. $\quad H_{\kappa} \stackrel{r}{\longrightarrow} H$, and $\lambda$ is an isolated eigenvalue of $H$ with finite multiplicity $m$; $J$ denotes an isolating interval for $\lambda, \mathscr{C l}$ the m-dimensional $\lambda$-eigenspace, and $P$ the eigenprojection $E[\lambda]$ onto $\mathscr{M}$.

Definition 2.4. Under Assumption 2.3, if $p$ is any real number $\geqq 0, \Sigma_{\kappa}(J)$ is concentrated to order $p$ provided that there are sets $C_{\kappa} \subset J$ such that $E_{\kappa}\left[C_{\kappa}\right] \stackrel{s}{\longrightarrow} P$ and $\nu\left(C_{\kappa}\right)=o\left(\kappa^{p}\right)$.

It turns out that $p$-th order concentration of $\Sigma_{\kappa}(J)$ hinges on the existence of a certain family of $p$-pairs for $H_{\kappa}$.

Definition 2.5. (cf. [2], p. 6) Under Assumption 2.3 suppose that $\left(\lambda_{j \kappa}, \varphi_{j \kappa}\right), j=1, \cdots, m$, are $p$-pairs for $H_{\kappa}$ such that $(1-P) \varphi_{j \kappa} \rightarrow 0$ for each $j$ and $\left(\varphi_{j \kappa}, \varphi_{k \kappa}\right) \rightarrow 0$ for $j \neq k$. Then $\left\{\varphi_{1 \kappa}, \cdots, \varphi_{m \kappa}\right\}$ will be called an asymptotic basis of order $p$ for $E_{\kappa}[J]$.

LEMMA 2.6. If $\left\{\varphi_{1 \kappa}, \cdots, \varphi_{m \kappa}\right\}$ is an asymptotic basis of order 0 for $E_{\kappa}[J]$, then the operator $P_{\kappa}=\sum_{j=1}^{m}\left(\cdot, \varphi_{j \kappa}\right) \varphi_{j \kappa} \stackrel{s}{\longrightarrow} P$, and the associated pseudoeigenvalues $\lambda_{\kappa} \rightarrow \lambda$.

Proof. The vectors $P \varphi_{j \kappa}=\varphi_{j \kappa}+o(1), j=1, \cdots, m$, satisfy

$$
\left(P \varphi_{j \kappa}, P \varphi_{k \kappa}\right)=\delta_{j \kappa}+o(1),
$$

and so can be orthonormalized to give a basis $\left\{\tilde{\varphi}_{1 \kappa}, \cdots, \tilde{\varphi}_{m k}\right\}$ of $\mathscr{M}$ such that $\left\|\varphi_{j \kappa}-\widetilde{\varphi}_{j \kappa}\right\|=o(1)$. Then for each $\varphi \in \mathscr{H}$,

$$
\begin{aligned}
P \varphi & =\sum_{j=1}^{m}\left(\varphi, \widetilde{\varphi}_{j \kappa}\right) \widetilde{\varphi}_{j \kappa}=\sum_{j=1}^{m}\left(\varphi, \varphi_{j \kappa}\right) \varphi_{j \kappa}+o(1) \\
& =P_{\kappa} \varphi+o(1),
\end{aligned}
$$

that is, $P_{\kappa} \stackrel{s}{\longrightarrow} P$.

As we have seen, Assumption 2.3 guarantees that $E_{\kappa[}\left[J^{\prime}\right] \stackrel{s}{\longrightarrow} P$ for any isolating interval $J^{\prime}$ about $\lambda$. Since $\varphi_{j \kappa}$ can be written, with 
an error $o(1)$, as a linear combination of a basis of $\mathscr{C}=P \mathscr{C}$, it follows that $E_{\kappa}\left[J^{\prime}\right] \varphi_{j \kappa}=\varphi_{j \kappa}+o(1), j=1, \cdots, m$. On the other hand, Lemma 1.3 shows that $\left(1-E_{\kappa}\left[\lambda_{\kappa}, o(1)\right]\right) \varphi_{j \kappa}=o(1), j=1, \cdots, m$, where the first $o(1)$ is suitably chosen as in the proof of Lemma 2.2. Combining these convergences, we obtain

$$
E_{\kappa}\left[J^{\prime}\right] \rho_{j \kappa}=E_{\kappa}\left[\lambda_{\kappa}, o(1)\right] \rho_{j \kappa}+o(1), j=1, \cdots, m .
$$

The norm of each side tends to 1 , which is impossible if the two projections $E_{\kappa}\left[J^{\prime}\right]$ and $E_{\kappa}\left[\lambda_{\kappa}, o(1)\right]$ remain orthogonal, that is, if the interval $\lambda_{\kappa}-o(1) \leqq \mu \leqq \lambda_{\kappa}+o(1)$ remains disjoint from $J^{\prime}$. Hence $\lambda_{\kappa}$ tends to $J^{\prime}$, and since $J^{\prime}$ can be any interval about $\lambda$, this proves $\lambda_{\kappa} \rightarrow \lambda$.

We are now in a position to prove the main

THEOREM 2.7. Under Assumption 2.3, $\Sigma_{\kappa}(J)$ is concentrated to order $p$ if and only if there is an asymptotic basis of order $p$ for $E_{\kappa}[J]$. In either case, the concentration sets $C_{\kappa}$ can be taken as the union of intervals of length $o\left(\kappa^{p}\right)$ centered on the corresponding pseudoeigenvalues $\lambda_{j \kappa}$.

Proof. Suppose that $\left\{\varphi_{1 \kappa}, \cdots, \varphi_{m \kappa}\right\}$ is an asymptotic basis of order $p$ for $E_{\kappa}[J]$, with associated pseudoeigenvalues $\lambda_{j \kappa}$. As in the proof of Lemma 2.2, Lemma 1.3 gives

$$
\varphi_{j \kappa}-E_{\kappa}\left[\lambda_{j \kappa}, o\left(\kappa^{p}\right)\right] \varphi_{j \kappa}=o(1), j=1, \cdots, m .
$$

Then with $C_{\kappa}=\bigcup_{j=1}^{m}\left\{\mu:\left|\lambda_{j \kappa}-\mu\right| \leqq o\left(\kappa^{p}\right)\right\}$ and $P_{\kappa}=\sum_{j=1}^{m}\left(\cdot, \varphi_{j \kappa}\right) \varphi_{j \kappa}$, we have $P_{\kappa}-E_{\kappa}\left[C_{\kappa}\right] P_{\kappa} \stackrel{s}{\longrightarrow} 0$. But from Lemma 2.6, $P_{\kappa} \stackrel{s}{\longrightarrow} P$. Hence if $\varphi \in \mathscr{M}$,

$$
\begin{aligned}
E_{\kappa}\left[C_{\kappa}\right] \varphi & =E_{\kappa}\left[C_{\kappa}\right] P \varphi \\
& =E_{\kappa}\left[C_{\kappa}\right]\left(P_{\kappa} \varphi+o(1)\right) \\
& =P_{\kappa} \varphi+o(1)+o(1) \\
& =P \varphi+o(1)+o(1)+o(1),
\end{aligned}
$$

where the uniform boundedness of the projections $E_{\kappa}\left[C_{\kappa}\right]$ has been used. Thus $E_{\kappa}\left[C_{\kappa}\right] \varphi \rightarrow P \varphi=\varphi$.

Since $\lambda_{j \kappa} \rightarrow \lambda$ by Lemma 2.6, $C_{\kappa} \subset J$ for small enough $\kappa$, and so $E_{\kappa}\left[C_{\kappa}\right] \leqq E_{\kappa}[J]$. Hence, if $\psi \perp \mathscr{M}$,

$$
\left\|E_{\kappa}\left[C_{\kappa}\right] \psi\right\| \leqq\left\|E_{\kappa}[J] \psi\right\| \longrightarrow\|P \psi\|=0 .
$$

But any vector $\theta \in \mathscr{H}$ is the sum of $\varphi=P \theta$ and $\psi=(1-P) \theta$, and we have shown that $E_{\kappa}\left[C_{\kappa}\right] \theta \rightarrow \varphi+0=P \theta$. Thus $E_{\kappa}\left[C_{\kappa}\right] \stackrel{s}{\longrightarrow} P$, and $\nu\left(C_{\kappa}\right)=o\left(\kappa^{p}\right)$, as desired. 
Conversely, suppose that $E_{\kappa}\left[C_{\kappa}\right] \stackrel{s}{\longrightarrow} P$, where $\nu\left(C_{\kappa}\right)=o\left(\kappa^{p}\right)$. For each $\kappa$, cover $C_{\kappa}$ with an open set $G_{\kappa}$ whose measure is within $\kappa^{p+1}$ of $\nu\left(C_{\kappa}\right) . \quad G_{\kappa}$ is the union of at most countably many disjoint open intervals $I_{\kappa}^{i}$, whose lengths are obviously $o\left(\kappa^{p}\right)$. Let

$$
P_{\kappa}^{i} \equiv E_{\kappa}\left[I_{\kappa}^{i}\right],
$$

and note that

$$
\sum_{i=1}^{\infty} P_{\kappa}^{i}=E_{\kappa}\left[G_{\kappa}\right] \geqq E_{\kappa}\left[C_{\kappa}\right] \text {. }
$$

The search for a $p$-pair $\left(\lambda_{\kappa}, \varphi_{\kappa}\right)$ will proceed in several steps.

I. Fix $\kappa>0$. Let $\mathscr{S} \equiv \mathscr{L} \cap\{\psi:\|\psi\|=1\} ; \mathscr{S}$ is compact since $\mathscr{C}$ is $m$-dimensional. For each $i=1,2, \cdots$, the function $\left\|P_{k}^{i}\right\|$ defined on $\mathscr{S}$ is continuous and so achieves a maximum, say at $\psi_{\kappa}^{i}$.

We claim that $\left\|P_{k}^{i} \psi_{k}^{i}\right\| \rightarrow 0$ as $i \rightarrow \infty$. For if not, there is a number $\varepsilon>0$ and an increasing sequence of integers $j$ for which $\left\|P_{k}^{j} \psi_{k}^{j}\right\|>\varepsilon$. Since the sequence $\psi_{k}^{j}$ lies in the unit ball of $\mathscr{A}$, it has a convergent subsequence, which we might as well denote with the same index $j$. Thus $\psi_{k}^{j} \rightarrow \psi_{k}$ as $j \rightarrow \infty$, with $\left\|\psi_{\kappa}\right\|=1, P \psi_{k}=\psi_{\kappa}$, and

$$
\left\|P_{\kappa}^{j} \psi_{\kappa}\right\|=\left\|P_{\kappa}^{j}\left(\psi_{\kappa}^{j}+o(1)\right)\right\| \geqq\left\|P_{k}^{j} \psi_{k}^{j}\right\|-o(1)>\varepsilon>0
$$

for large $j$. Hence $1=\left\|\psi_{\kappa}\right\|^{2} \geqq \sum_{j=1}^{\infty}\left\|P_{\kappa}^{j} \psi_{\kappa}^{i}\right\|^{2}=\infty$; absurd.

II. Since $1 \geqq\left\|P_{\kappa}^{i} \psi_{\kappa}^{i}\right\| \rightarrow 0$ as $i \rightarrow \infty$, there is for each $\kappa$ at least one integer $i(\kappa)$ such that

$$
\begin{aligned}
P_{\kappa}^{i(\kappa)} \psi_{\kappa}^{i(\kappa)} & =\max \left\{\left\|P_{\kappa}^{i} \psi_{\kappa}^{i}\right\|: i=1,2, \cdots\right\} \\
& =\max \left\{\left\|P_{\kappa}^{i} \psi\right\|: i=1,2, \cdots, \psi \in \mathscr{S}\right\} .
\end{aligned}
$$

For each $\kappa$, set $Q_{\kappa} \equiv P_{\kappa}^{i(\kappa)}=E_{\kappa}\left[I_{\kappa}^{i(\kappa)}\right]$ and $\psi_{\kappa} \equiv \psi_{\kappa}^{i(\kappa)}$.

III. Letting $\kappa$ vary, we claim that

$$
\left\|Q_{\kappa} \psi_{\kappa}\right\| \geqq\left(\frac{1}{2(m+2)}\right)^{1 / 2} \equiv \varepsilon>0
$$

for all sufficiently small $\kappa$. For, if not, there is a sequence $\kappa_{n} \rightarrow 0$ such that $Q_{\kappa_{n}} \psi_{\kappa_{n}}$ violates (1) for all $n=1,2, \ldots$. Since the sequence $\psi_{n} \equiv \psi_{\kappa_{n}}$ lies in the unit ball of $\mathscr{C}$, it has a subsequence, again denoted by $\psi_{n}$, converging to some $\psi \in \mathscr{M}$. By the definition of $\psi_{n}=\psi_{\kappa_{n}}=\psi_{\kappa_{n}}^{i\left(\kappa_{n}\right)}$ we have, uniformly in $i=1,2, \cdots$,

$$
\begin{aligned}
\left\|P_{\kappa_{n}}^{i} \psi\right\|^{2} & =\| P_{\kappa_{n}}^{i}\left(\psi_{n}+o(1) \|^{2}\right. \\
& =\left\|P_{\kappa_{n}}^{i} \psi_{n}\right\|^{2}+o(1) \\
& <\frac{1}{2(m+2)}
\end{aligned}
$$


for $n$ large enough. On the other hand, $E_{\kappa}\left[C_{\kappa}\right] \psi \rightarrow \psi$ by hypothesis, so that $\left\|E_{\kappa_{n}}\left[C_{\kappa_{n}}\right] \psi\right\|^{2}>(m+1) /(m+2)$ for sufficiently large $n$. Fix any such $n$. We have $\left\|E_{\kappa_{n}}\left[C_{\kappa_{n}}\right] \psi\right\|^{2} \leqq \lim _{q \rightarrow \infty} \sum_{i=1}^{q}\left\|P_{\kappa_{n}}^{i} \psi\right\|^{2}$; and so for sufficiently large $q, \sum_{i=1}^{q}\left\|P_{\kappa_{n}}^{i} \psi\right\|^{2}>(m+1) /(m+2)$. Thus with $n$ still fixed we can define integers

$$
q_{j}=\max \left\{q: \sum_{i=1}^{q}\left\|P_{\kappa_{n}}^{i} \psi\right\|^{2} \leqq \frac{j}{m+2}\right\},
$$

for $j=1, \cdots, m+1$, and $q_{0}=0$. These integers satisfy

$$
q_{0}<q_{1}<\cdots<q_{m+1} \text { : for if } q_{j}=q_{j+1}=q
$$

for some $j$, then

$$
\sum_{i=1}^{q}\left\|P_{\kappa_{n}}^{i} \psi\right\|^{2} \leqq \frac{j}{m+2}, \sum_{i=1}^{q+1}\left\|P_{\kappa_{n}}^{i} \psi\right\|^{2}>\frac{j+1}{m+2}
$$

by the definition (3), and so $\left\|P_{\kappa_{n}}^{q+1} \psi\right\|^{2}>1 /(m+2)$, contradicting (2). Thus we can set

$$
S_{n}^{j} \equiv \sum_{i=q_{j-1}+1}^{p_{j}} P_{\kappa_{n}}^{i}, j=1,2, \cdots, m+1 .
$$

In view of the pairwise disjointness of the intervals $I_{\kappa_{n}}^{i}$ for different $i$, the $S_{n}^{j}, j=1, \cdots, m+1$, are pairwise orthogonal projections for each fixed $n$. Moreover,

$$
\left\|S_{n}^{j} \psi\right\|>\frac{1}{2} \frac{1}{m+2}, \quad j=1, \cdots, m+1 .
$$

For if, e.g., $\left\|S_{n}^{1} \psi\right\| \leqq(1 / 2) 1 /(m+2)$, then using (2) we have

$$
\begin{aligned}
\sum_{i=1}^{q_{1}+1}\left\|P_{\kappa_{n}}^{i} \psi\right\|^{2} & =\left\|S_{n}^{1} \psi\right\|^{2}+\left\|P_{\kappa_{n}}^{q_{1}+1} \psi\right\|^{2} \\
& <\frac{1}{2} \frac{1}{m+2}+\frac{1}{2} \frac{1}{m+2}=\frac{1}{m+2}
\end{aligned}
$$

contradicting the definition of $q_{1}$ in (3). Hence (4) holds.

Now we have $m+1$ sequences $\left\{\psi_{j n}=S_{n}^{j} \psi, n=1,2, \cdots\right\}, j=$ $1, \cdots, m+1$, where $\left\|\psi_{j k}\right\| \geqq \varepsilon>0$ and $\left(\psi_{j n}, \psi_{k n}\right)=0$ for $j \neq k$. Moreover, $\psi_{j n}=E_{\kappa_{n}}[J] \psi_{j n}$ for each $j$, and since $E_{\kappa_{n}}[J] \stackrel{s}{\longrightarrow} P$, this means $(1-P) \psi_{j n} \rightarrow 0$. Then Lemma 2.8 (below) implies that $\operatorname{dim} \mathscr{C l} \geqq$ $m+1$. This contradiction establishes (1).

IV. We assert that $\left\|Q_{\kappa} \psi_{\kappa}\right\| \rightarrow 1$ as $\kappa \rightarrow 0$. For if not, then there is a number $\delta>0$ and a sequence $\kappa_{n} \rightarrow 0$ such that $\left\|Q_{\kappa_{n}} \psi_{\kappa_{n}}\right\|<1-\delta$ for all $n=1,2, \cdots$. Let $Q_{n} \equiv Q_{\kappa_{n}}, \psi_{n} \equiv \psi_{\kappa_{n}}$. The sequence 


$$
\psi_{n}^{\prime} \equiv P Q_{n} \psi_{n}
$$

lies in the unit ball of $\mathscr{K}$, and so by passing to a subsequence we can assume that $\psi_{n}^{\prime} \rightarrow$ some $\psi^{\prime}$. Since $P \psi_{n}=\psi_{n}$ and $\left\|\psi_{n}\right\|=1$,

$$
\begin{aligned}
\left\|\psi_{n}^{\prime}\right\| \equiv\left\|P Q_{n} \psi_{n}\right\| & \geqq\left|\left(P Q_{n} \psi_{n}, \psi_{n}\right)\right| \\
& =\left|\left(Q_{n} \psi_{n}, \psi_{n}\right)\right| \\
& =|| Q_{n} \psi_{n} \|^{2}>\varepsilon^{2}>0
\end{aligned}
$$

in view of (1), part III. Thus $\psi^{\prime} \neq 0$.

On the other hand, $Q_{n} \leqq E_{\kappa_{n}}[J] \stackrel{s}{\longrightarrow} P$ gives $(1-P) Q_{n} \stackrel{s}{\longrightarrow} 0$, and since the $\psi_{n}$ are confined to the finite-dimensional space $\mathscr{C}$, this gives

$$
\begin{aligned}
Q_{n} \psi_{n} & =P Q_{n} \psi_{n}+(1-P) Q_{n} \psi_{n} \\
& =P Q_{n} \psi_{n}+o(1)
\end{aligned}
$$

that is,

$$
Q_{n} \psi_{n}=\psi_{n}^{\prime}+o(1)=\psi^{\prime}+o(1)
$$

Hence

$$
\begin{aligned}
Q_{n} \psi^{\prime} & =Q_{n}\left(Q_{n} \psi_{n}+o(1)\right)=Q_{n}^{2} \psi_{n}+o(1) \\
& =Q_{n} \psi_{n}+o(1) \\
& =\psi^{\prime}+o(1)+o(1)
\end{aligned}
$$

Since $\psi^{\prime} \neq 0$ we may set $\psi=\left\|\psi^{\prime}\right\|^{-1} \psi^{\prime}$, and now

$$
\begin{aligned}
\left\|Q_{n} \psi\right\| & =\|\psi+o(1)\|=1+o(1)>1-\delta \\
& >\left\|Q_{n} \psi_{n}\right\|
\end{aligned}
$$

for large $n$. But $\psi \in \mathscr{S}$, and the last inequality contradicts the maximality of $\left\|Q_{\kappa_{n}} \psi_{\kappa_{n}}\right\|$ (part I). Thus $\left\|Q_{\kappa} \psi_{\kappa}\right\| \rightarrow 1$ is proved.

V. Let $\varphi_{\kappa} \equiv\left\|Q_{\kappa} \psi_{\kappa}\right\|^{-1} Q_{\kappa} \psi_{\kappa}$. Then $\left\|\varphi_{\kappa}\right\|=1$. Recalling that $Q_{\kappa} \equiv E_{\kappa}\left[I_{\kappa}^{i(\kappa)}\right]$, we choose $\lambda_{\kappa} \equiv$ midpoint of $I_{\kappa}^{i(\kappa)}$. Since the measure generated by $\left\|E_{\kappa}(\mu) \varphi_{\kappa}\right\|^{2}$ is supported in $I_{\kappa}^{i(\kappa)}$, an interval of length $o\left(\kappa^{p}\right)$, the spectral formula yields the estimate $\left\|\left(H_{\kappa}-\lambda_{\kappa}\right) \varphi_{\kappa}\right\|=o\left(\kappa^{p}\right)$; and we have a $p$-pair $\left(\lambda_{\kappa}, \varphi_{\kappa}\right)$ for $H_{\kappa}$ with $(1-P) \varphi_{\kappa} \rightarrow 0$.

VI. To find another $p$-pair we repeat the whole procedure. First put $\lambda_{1 \kappa} \equiv \lambda_{\kappa}, \varphi_{1 \kappa} \equiv \varphi_{\kappa}, \psi_{1 \kappa} \equiv \psi_{\kappa}$ and $Q_{1 \kappa} \equiv Q_{\kappa}$, and note that, since $\left\|\psi_{1 \kappa}\right\|=1$, part IV gives

$$
Q_{1 \kappa} \psi_{1 \kappa}=\psi_{1 \kappa}+o(1) .
$$

For each $\kappa$, let $\mathscr{C}_{2 \kappa} \equiv\left\{\psi \in \mathscr{L}, \psi \perp \psi_{1 k}\right\}$ and $\mathscr{S}_{2 \kappa}=\left\{\psi \in \mathscr{C l}_{2 \kappa}:\|\psi\|=1\right\}$. Since $\mathscr{S}_{2 \kappa}$ is compact for each $\kappa$, the arguments of I, II, and III apply without change, to show that $\left\|Q_{\kappa} \psi_{\kappa}\right\|>\varepsilon$, where now 


$$
\left\|Q_{\kappa} \psi_{\kappa}\right\|=\max \left\{\left\|P_{\kappa}^{i} \psi\right\|: i=1,2, \cdots, \psi \in \mathscr{S}_{2 \kappa}\right\} .
$$

Note that $\psi_{\kappa} \perp \psi_{1 \kappa}$ but that $Q_{\kappa}$ may be the same as $Q_{1 \kappa}$ for some or all $\kappa$.

The argument in IV must be adjusted slightly. Denote by $P_{\kappa}$ the projection on $\mathscr{C}_{2 \kappa}$, and put $P_{n}=P_{\kappa_{n}}$, where $\kappa_{n} \rightarrow 0$ is the sequence in IV. Instead of (5), set

$$
\psi_{n}^{\prime}=P_{n} Q_{n} \psi_{n}
$$

and put $\psi^{\prime}=$ limit (subsequence) $\psi_{n}^{\prime}$ as before. Since $P_{n} \psi_{n}=\psi_{n}$, inequality (6) holds with $P_{n}$ in place of $P$. Relation (7) holds without change, but in order to get (8) we need in addition that $\left(P-P_{n}\right) Q_{n} \psi_{n}=$ $o(1)$. This follows from (9) together with the fact that $P_{\kappa}^{j} \perp P_{\kappa}^{j}$ for $i \neq j$ :

$$
\begin{aligned}
\left(Q_{n} \psi_{n}, \psi_{1 n}\right) & =\left(Q_{n} \psi_{n}, Q_{1 n} \psi_{1 n}\right)+o(1) \\
& =\left\{\begin{array}{l}
0+o(1) \text { if } Q_{n} \neq Q_{1 n} \\
\left(\psi_{n}, Q_{1 n}^{2} \psi_{1 n}\right)=\left(\psi_{n}, Q_{1 n} \psi_{1 n}\right) \text { if } Q_{n} \neq Q_{1 n}
\end{array}\right. \\
& =\left\{\begin{array}{l}
o(1) \\
\left(\psi_{n}, \psi_{1 n}\right)+o(1)
\end{array}\right\}=o(1) .
\end{aligned}
$$

Thus the projection of $Q_{n} \psi_{n}$ along $\psi_{1 n}$, i.e. $\left(P-P_{n}\right) Q_{n} \psi_{n}$, tends to 0 . Hence (8) holds with $\psi_{n}^{\prime}$ given by $\left(5^{\prime}\right)$, and it follows as before that $Q_{n} \psi^{\prime}=\psi^{\prime}+o(1)$. Again we set $\psi=\left\|\psi^{\prime}\right\|^{-1} \psi^{\prime}$, and it follows that $Q_{n} \psi=\psi+o(1)$. But $\psi$ is, like $\psi^{\prime}$, the limit of vectors in $\mathscr{C}_{2 \kappa_{n}}$, and so $P_{n} \psi=\psi+o(1)$. Hence

$$
\begin{aligned}
\left\|Q_{n} P_{n} \psi\right\| & =\left\|Q_{n} \psi+o(1)\right\|=\|\psi+o(1)+o(1)\| \\
& >1-\delta>\left\|Q_{n} \psi_{n}\right\|
\end{aligned}
$$

for large $n$, contradicting the choice of $\psi_{n}$ as a vector where $\left\|Q_{n} \psi_{n}\right\|$ is a maximum among $\psi \in \mathscr{S}_{2 \kappa_{n}}$. Thus $\left\|Q_{n} \psi_{n}\right\|<1-\delta$ is untenable, and $\left\|Q_{n} \psi_{n}\right\| \rightarrow 1$ as befere.

Part $\mathrm{V}$ goes through without change, and we have constructed a second $p$-pair $\left(\lambda_{\kappa}, \varphi_{k}\right)$ with $(1-P) \varphi_{\kappa} \rightarrow 0$. Denoting this second batch of quantities $\lambda_{\kappa}, \varphi_{\kappa}, \psi_{\kappa}$ and $Q_{\kappa}$ by $\lambda_{2 \kappa}, \varphi_{2 \kappa}, \psi_{2 \kappa}$, and $Q_{2 \kappa}$, and bearing in mind that $\left\|Q_{\kappa} \psi_{k}\right\| \rightarrow 1$, we see that

$$
Q_{2 \kappa} \psi_{2 \kappa}=\psi_{2 \kappa}+o(1) .
$$

Hence

$$
\begin{aligned}
\left(\varphi_{2 \kappa}, \varphi_{1 \kappa}\right) & =\left(Q_{2 \kappa} \psi_{2 \kappa}, Q_{1 \kappa} \psi_{1 \kappa}\right)+o(1) \\
& =\left(\psi_{2 \kappa}, \psi_{1 \kappa}\right)+o(1)+o(1) \\
& =o(1)
\end{aligned}
$$


since $\psi_{2 \kappa} \perp \psi_{1 \kappa}$ by construction.

It is clear that this procedure can be repeated $m-2$ more times. At the $k$ th stage, steps (10) and (11) can be verified with the index 1 replaced by each $j<k$ and 2 replaced by $k$. After $m$ iterations we have constructed the required asymptotic basis $\left\{\varphi_{1 \kappa}, \cdots, \varphi_{m \kappa}\right\}$ for $E_{\kappa}[J]$. Note that the procedure halts here since there are no unit vectors in tl perpendicular to all of $\psi_{1 \kappa}, \cdots, \psi_{m \kappa}$.

Of course it follows from the first part of the theorem that $\Sigma_{\kappa}(J)$ is concentrated on the union of the (at most) $m$ intervals $I_{\kappa}^{i(\kappa)}$ whose midpoints were chosen as the $\lambda_{j \kappa}$. This completes the proof of the theorem.

In the proof, the following fact was used:

Lemma 2.8. Let $P$ be a projection of dimension $m$. Let

$$
\psi_{1 n}, \cdots, \psi_{d n}, n=1,2, \cdots
$$

be sequences of vectors satisfying $(1-P) \psi_{j n} \rightarrow 0,1 \geqq\left\|\psi_{j_{n}}\right\| \geqq \varepsilon>0$, and $\left(\psi_{j n}, \psi_{k m}\right) \rightarrow 0$ for $j \neq k$. Then $d \leqq m$.

Proof. $P \psi_{1 n}$ is a sequence in the unit ball of the $m$-dimensional space $P \mathscr{H}$. By passing to a subsequence, we can therefore assume that $P \psi_{1 n} \rightarrow \psi_{1}$ for some vector $\psi_{1}$ with $P \psi_{1}=\psi_{1}$. Since $(1-P) \psi_{1 n} \rightarrow 0$,

$$
\psi_{1 n}=P \psi_{1 n}+(1-P) \psi_{1 n}=\psi_{1}+o(1)
$$

as $n \rightarrow \infty$. Moreover $\left\|\psi_{1 n}\right\| \geqq \varepsilon$ gives $\psi_{1} \neq 0$. Looking at the corresponding subsequence of $\psi_{2 n}$, we can apply the same argument to find $\psi_{2} \neq 0$ with $P \psi_{2}=\psi_{2}$ and $\psi_{2 n} \rightarrow \psi_{2}$. Also,

$$
\left(\psi_{1}, \psi_{2}\right)=\left(\psi_{1 n}+o(1), \psi_{2 n}+o(1)\right)=o(1)+o(1),
$$

whence $\psi_{1} \perp \psi_{2}$. In this fashion we can construct pairwise orthogonal nonzero vectors $\psi_{1}, \cdots, \psi_{d}$ in $P \mathscr{H}$. Hence $\operatorname{dim} P \geqq d$.

The "only if" part of Theorem 2.7 will not be used in what follows.

CoROllary 2.9. Under Assumption 2.3, if $\left\{\varphi_{1 \kappa}, \cdots, \varphi_{m \kappa}\right\}$ and $\left\{\Phi_{1 \kappa}, \cdots, \Phi_{m \kappa}\right\}$ are two asymptotic bases of order $p$ for $E_{\kappa}[J]$, then the corresponding pseudoeigenvalues $\lambda_{i \kappa}$ and $\Lambda_{j \kappa}$ satisfy

$$
\Lambda_{j \kappa}=\lambda_{j \kappa}+o\left(\kappa^{p}\right), \quad j=1, \cdots, m
$$

(possibly after the $\Lambda_{j \kappa}$ have been re-indexed).

Proof. By Theorem 2.7, $\Sigma_{\kappa}(J)$ is concentrated on the union $C_{\kappa}$ of open intervals of length $o\left(\kappa^{p}\right)$ about the $\lambda_{j \cdot \kappa}$. Further, in view of Lemma 1.3, by lengthening the component intervals of $C_{\kappa}$ (still keep- 
ing them $o\left(\kappa^{p}\right)$ in length), we may assume that $\left(1-E_{\kappa}\left[I_{\kappa}^{(i)}\right]\right) \varphi_{i \kappa}=o(1)$, where $I_{\kappa}^{i}$ is the component subinterval of $C_{\kappa}$ containing $\lambda_{i \kappa}$. On the other hand, for each $j=1, \cdots, m$, since $(1-P) \Phi_{j k} \rightarrow 0$ and $P$ is $m$ dimensional, there is no sequence $\kappa_{n} \rightarrow 0$ for which $\Phi_{j \kappa_{n}} \stackrel{w}{\longrightarrow} 0$. Then Lemma 1.4 shows that each $A_{j \kappa}$ is within $o\left(\kappa^{p}\right)$ of $C_{\kappa}$. By again lengthening the components of $C_{\kappa}$ (still keeping them $o\left(\kappa^{p}\right)$ in length) we can assume that $A_{j \kappa} \in C_{\kappa}, j=1, \cdots, m$, and (by Lemma 1.3) that $\left(1-E_{\kappa}\left[I_{\kappa}^{j}\right]\right) \Phi_{j \kappa}=o(1)$, where $I_{\kappa}^{j}$ is the component interval of $C_{\kappa}$ containing $\Lambda_{j \kappa}$.

Let the distinct open component intervals of the adjusted $C_{\kappa}$ be numbered $I_{\kappa}^{1}, \cdots, I_{\kappa}^{s_{\kappa}}, s_{\kappa} \leqq m$. Each $I_{\kappa}^{i}$ is still of length $o\left(\kappa^{p}\right)$, so the asserted relabelling of the $\Lambda_{j \kappa}$ could be carried out provided the same number of $\Lambda_{j \kappa}$ 's as $\lambda_{i \kappa}$ 's belong to $I_{\kappa}^{k}$.

To see that this must be the case, at least for sufficiently small $\kappa$, we suppose the contrary, i.e. that for some sequence $\kappa_{n} \rightarrow 0$ the component interval $I_{\kappa_{n}}^{k_{n}}$ contains $\lambda_{i \kappa_{n}}$ for $m\left(k_{n}\right)$ different $i$ and contains $\Lambda_{j \kappa_{n}}$ for $M\left(k_{n}\right)>m\left(k_{n}\right)$ different $j$. Then for each $n$, the $M\left(k_{n}\right)$ different pseudoeigenvectors $\Phi_{j \kappa_{n}}$, together with the $m-m\left(k_{n}\right)$ different pseudoeigenvectors $\varphi_{j \kappa_{n}}$ not associated with the $\lambda_{i \kappa_{n}}$ in $I_{\kappa_{n}}^{k_{n}}$, make up a family $\left\{\psi_{1 n}, \cdots, \psi_{d n}\right\}$, where $d=m-m\left(k_{n}\right)+M\left(k_{n}\right)>m$. By the construction of the intervals $I_{n}^{k}$ it is clear that $\left\|\psi_{j n}\right\| \rightarrow 1$ and

$$
\left(\psi_{j \kappa}, \psi_{i \kappa}\right) \longrightarrow 0 \text { if } i \neq j
$$

and of course $(1-P) \psi_{j k} \rightarrow 0$ by definition. Thus $d>m$ contradicts Lemma 2.8, and the asserted relabelling can be carried out.

CoRollary 2.10. Under Assumption 2.3, $\Sigma_{\kappa}(J)$ is concentrated to order 0 .

Proof. Let $\left\{\varphi_{1}, \cdots, \varphi_{m}\right\}$ be an orthonormal basis for Ml. Application of Lemma 2.2 to each $\varphi_{j}$ in turn yields an asymptotic basis of order 0 for $E_{\kappa}[J]$, and the assertion follows from Theorem 2.7.

It should be noted that Corollary 2.10 can fail if $\lambda$ has infinite multiplicity.

3. Construction of asymptotic bases of order $p>0$. Corollary 2.10 required nothing beyond Assumption 2.3, but to obtain higherorder asymptotic bases we shall apply the formal perturbation method, and this requires that $H_{\kappa}$ have a certain form.

Suppose that $V$ is a symmetric operator in $\mathscr{C}$ such that some linear set $\mathscr{D} \subset \mathscr{D}(H) \cap \mathscr{D}(V)$ is dense; then $H+\kappa V$ is defined and symmetric on $\mathscr{D}$. We shall suppose that for sufficiently small $\kappa,(H+\kappa V) \mid \mathscr{D}$ admits a self-adjoint extension. Then $H_{\kappa}$ is taken to 
be one such extension. Specifically, we consider two cases:

Assumption 3.1. At least one of the following holds:

(a) $H_{\kappa}$ is any self-adjoint extension of $H+\kappa V$, and the restriction of $H$ to $\mathscr{D}$ is essentially self-adjoint; or

(b) $(H \varphi, \varphi) \geqq(\varphi, \varphi)$ and $(V \varphi, \varphi) \geqq 0$ for all $\varphi \in \mathscr{D}, H_{\kappa}$ is the Friedrichs extension of $H+\kappa V$, and the restriction of $H$ to $\mathscr{D}$ has $H$ as its Friedrichs extension;

and it is still assumed that $\lambda$ is an isolated eigenvalue of $H$ with multiplicity $m$.

In view of Lemma 2.1, either (a) or (b) guarantees that $H_{\kappa} \stackrel{r}{\longrightarrow} H$, and so Assumption 2.3 is satisfied and all the results of $\S 2$ follow under Assumption 3.1.

We consider numbers $\lambda_{\kappa}$ and vectors $\varphi_{\kappa}$ of the form

$$
\begin{aligned}
& \lambda_{\kappa}=\lambda+\kappa \lambda^{(1)}+\cdots+\kappa^{p} \lambda^{(p)} \\
& \psi_{\kappa}=\psi^{(o)}+\kappa \psi^{(1)}+\cdots+\kappa^{p} \psi^{(p)}
\end{aligned}
$$

where $p$ is an integer $\geqq 1$ and $\psi^{(0)}$ is an eigenvector of $H$ associated with $\lambda$. If $\psi^{(0)}, \psi^{(1)}, \cdots, \psi^{(p)} \in \mathscr{D}$, then $\left(H_{\kappa}-\lambda_{\kappa}\right) \psi_{\kappa}$ can be computed from the formulas $H_{\kappa} \psi^{(r)}=H \psi^{(r)}+\kappa V \psi^{(r)}$. We find

$$
\begin{aligned}
\left(H_{\kappa}-\lambda_{\kappa}\right) \psi_{\kappa} & =\sum_{r=1}^{p} C^{(r)} \kappa^{r}+O\left(\kappa^{p+1}\right), \\
C^{(r)} & =(H-\lambda) \psi^{(r)}+B^{(r)}, \\
B^{(r)} & =\left(V-\lambda^{(1)}\right) \psi^{(r-1)}-\sum_{k=0}^{r-2} \lambda^{(r-k)} \psi^{(k)},
\end{aligned}
$$

where in $B^{(1)}$ the formal term $\sum_{k=0}^{-1}$ is to be suppressed. In order that $\left(\lambda_{\kappa}, \varphi_{\kappa}=\|\psi\|^{-1} \psi_{\kappa}\right)$ be a $p$-pair for $H_{\kappa}$, it is necessary and sufficient that $C^{(r)}=0, r=1,2, \cdots, p$.

For simplicity we consider first the case where $\lambda$ is simple, i.e. $m=1$. Then $P \varphi=\left(\varphi, \psi^{(0)}\right) \psi^{(0)}$ for any $\varphi \in \mathscr{D}$. If $\lambda^{(k)}$ and $\psi^{(k)}$ have already been chosen for $k<r$, then

$$
\begin{aligned}
P B^{(r)}= & \left(\left[\left(V-\lambda^{(1)} \psi^{(r-1)}\right)-\sum_{k=1}^{r-2} \lambda^{(r-k)} \psi^{(k)}\right] \psi^{(o)}\right) \psi^{(o)} \\
& -\lambda^{(r)} \psi^{(o)}
\end{aligned}
$$

can be made to vanish by choosing

$$
\left.\lambda^{(r)}=\left(\left[\left(V-\lambda^{(1)}\right) \psi^{(r-1)}\right)-\sum_{k=1}^{r-2} \lambda^{(r-k)} \psi^{(k)}\right], \psi^{(o)}\right) .
$$

Since $(H-\lambda) P=0$ as well, we now have

$$
C^{(r)}=(H-\lambda)(1-P) \psi^{(r)}+(1-P) B^{(r)} .
$$


Recalling that the reduced resolvent $S=\int_{\text {off } J}(\mu-\lambda)^{-1} d E(\mu)$ of $H$ at $\lambda$ is bounded on $\mathscr{C}$ and satisfies $(H-\lambda) S=1-P, S P=P S=0$, we choose $\psi^{(r)}$ by

$$
(1-P) \psi^{(r)}=-S B^{(r)}, P \psi^{(r)} \text { arbitrary, }
$$

so that (16) vanishes, as desired.

Formulas (15) and (17) are meaningful if and only if $V \psi^{(r-1)}$ is defined. By induction, then, these choices can be made for $r=1, \cdots, p$ if and only if $\psi^{(0)}$ lies in the domain of each of the composite operators $T^{1} \cdots T^{p}$, where each $T^{j}$ is either $S$ or $S V$.

This condition guarantees that $\lambda^{(r)}$ and $\psi^{(r)}$ are defined and $C^{(r)}=0, r=1, \cdots, p$, and that $\psi^{(r)} \in \mathscr{D}, r=0, \cdots, p-1$. However it does not guarantee that $\psi^{(p)} \in \mathscr{D}$. Thus the computation leading to (13) is not quite justified, and we must modify $\psi^{(p)}$ as in Lemma 2.2. Letting

$$
\begin{aligned}
\psi_{\kappa}^{(p)} & =\left(H_{\kappa}-i\right)^{-1}(H-i) \psi^{(p)} \\
\psi_{\kappa}^{\prime} & =\psi^{(0)}+\kappa \psi^{(1)}+\cdots+\kappa^{p-1} \psi^{(p-1)}+\kappa^{p} \psi_{\kappa}^{(p)},
\end{aligned}
$$

and using the fact that $C^{(r)}=0$ for $r=1, \cdots, p$, we find

$$
\left(H_{\kappa}-\lambda_{\kappa}\right) \psi_{\kappa}^{\prime}=\kappa^{p}(\lambda-i)\left(\psi^{(p)}-\psi_{\kappa}^{(p)}\right)+O\left(\kappa^{p+1}\right),
$$

which is $o\left(\kappa^{p}\right)$ since $\psi_{\kappa}^{(p)} \rightarrow \psi^{(p)}$. Hence $\left(\lambda_{\kappa}, \varphi_{\kappa}=\left\|\psi_{\kappa}^{\prime}\right\|^{-1} \psi_{k}^{\prime}\right)$ is a $p$-pair for $H_{\kappa}$, and since $\varphi_{\kappa} \rightarrow \psi^{(0)}$, the singleton $\left\{\varphi_{\kappa}\right\}$ is an asymptotic basis of order $p$ for $E_{\kappa}[J]$.

If $\lambda$ is degenerate $(m>1)$ we seek numbers $\lambda_{j \kappa}$ and vectors $\psi_{j \kappa}$ of the form (12), for $j=1, \cdots, m$, such that the quantities $C_{j}^{(r)}$ as in (13) vanish for $r=1, \cdots, p$. Now (14) no longer holds, and indeed $P B_{j}^{(r)}$ cannot be made to vanish merely by the choice of $\lambda_{j}^{(r)}$.

Consider first the case $p=1$. Assume $\mathscr{C} \subset \mathscr{D}(V)$. Since $\psi_{j}^{(o)} \in \mathscr{L}$, we have

$$
P B_{j}^{(1)}=P\left(V-\lambda_{j}^{(1)}\right) \psi_{j}^{(0)}=P\left(V-\lambda_{j}^{(1)}\right) P \psi_{j}^{(0)} .
$$

The $m$-dimensional symmetric operator $P V P$ has $s \leqq m$ eigenvalues $\mu_{1}<\cdots<\mu_{s}$ and corresponding orthogonal eigenprojections $Q_{1}, \cdots, Q_{s}$ :

$$
P=\sum_{i=1}^{s} Q_{i}, P V P=\sum_{i=1}^{s} \mu_{i} Q_{i} .
$$

Put $m_{i}=\operatorname{dim} Q_{i}$, so that $\sum_{i=1}^{s} m_{i}=m$. For each $j=1, \cdots, m$ put $i(j)=$ least $i$ such that $j \leqq m_{1}+\cdots+m_{i}$; of course in general more than one $j$ will correspond to a given $i(j)$. Let $P_{j}^{(1)}=Q_{i(j)}$, and choose 


$$
\begin{aligned}
\lambda_{j}^{(1)} & =\mu_{i(j)} \\
\dot{\psi}_{j}^{(o)} & =P_{j}^{(1)} \psi_{j}^{(o)},\left(\psi_{j}^{(0)}, \psi_{k}^{(o)}\right)=\delta_{j k} .
\end{aligned}
$$

Then, with $V^{(1)} \equiv V$, we have

$$
P\left(V^{(1)}-\lambda_{j}^{(1)}\right) P_{j}^{(1)}=P_{j}^{(1)}\left(V^{(1)}-\lambda_{j}^{(1)}\right) P=0,
$$

so that (19) causes (18) to vanish for each $j=1, \cdots, m$. Now $C_{j}^{(1)}$ is given by (16) with $r=1$, and $\psi_{j}^{(1)}$ can be chosen as in (17) with $r=1$ to complete the annihilation of $C_{j}^{(1)}$. The nonuniqueness of the $\psi_{j}^{(0)}$ with a fixed $i(j)$ in (19) and of the $P \psi_{j}^{(1)}$ in (17) should be borne in mind.

In the choices for $r=2$, we shall need the reduced resolvent $S_{j}^{(1)}=\sum_{i \neq i(j)}\left(\mu_{i}-\lambda_{j}^{(1)}\right)^{-1} Q_{i}$ of $P V^{(1)} P$ at $\lambda_{j}^{(1)}$, which satisfies

$$
P\left(V^{(1)}-\lambda_{j}^{(1)}\right) S_{j}^{(1)}=P-P_{j}^{(1)}, S_{j}^{(1)} P_{j}^{(1)}=P_{j}^{(1)} S_{j}^{(1)}=0 .
$$

Passing now to the case $p=2$, we assume that $\mathscr{C} \subset \mathscr{D}\left(V^{(1)} S V^{(1)}\right)$. This certainly insures that $\mathscr{C l} \subset \mathscr{D}\left(V^{(1)}\right)$, so it can be supposed that the $\lambda_{j}^{(1)}, \psi_{j}^{(0)}$, and $\psi_{j}^{(1)}$ have been chosen as above to make the $C_{j}^{(1)}$ vanish. In studying the $C_{j}^{(2)}$ we treat simultaneously all the $j$ with a given $i(j)$, and for any such $j$ we consider successively the components $P_{j}^{(1)} B_{j}^{(2)}$ and $\left(P-P_{j}^{(1)}\right) B_{j}^{(2)}$ of $P B_{j}^{(2)}$. In view of (17) with $r=1$, (19), and (20), the first of these components is

$$
P_{j}^{(1)} B_{j}^{(2)}=P_{j}^{(1)}\left(V_{j}^{(2)}-\lambda_{j}^{(2)}\right) P_{j}^{(1)} \psi_{j}^{(0)},
$$

where $V_{j}^{(2)} \equiv\left(V^{(1)}-\lambda_{j}^{(1)}\right)(-S)\left(V^{(1)}-\lambda_{j}^{(1)}\right)$ is, by our assumption, defined at least on $\mathscr{M}$. Notice that this would not be true without the second-order assumption on $\mathscr{C}$. Now the same arguments can be applied to $P_{j}^{(1)} V_{j}^{(2)} P_{j}^{(1)}$ as were used on $P V^{(1)} P$ following (18). We are led to define, for each $j$, a projection $P_{j}^{(2)} \leqq P_{j}^{(1)}$ and a number $\lambda_{j}^{(2)}$ such that $P_{j}^{(1)}\left(V_{j}^{(2)}-\lambda_{j}^{(2)}\right) P_{j}^{(2)}=P_{j}^{(2)}\left(V_{j}^{(2)}-\lambda_{j}^{(2)}\right) P_{j}^{(1)}=0$. Then the choice

$$
\psi_{j}^{(0)}=P_{j}^{(2)} \psi_{j}^{(0)},\left(\psi_{j}^{(0)}, \psi_{k}^{(0)}\right)=\delta_{j k}
$$

is possible and causes (22) to vanish.

The second component of $P B_{j}^{(2)}$ is annihilated by refining the choice of $\psi_{j}^{(1)}$. In view of (17) with $r=1,(20)$, and the definition of $V_{j}^{(2)}$,

$$
\begin{aligned}
\left(P-P_{j}^{(1)}\right) B_{j}^{(2)}= & \left(P-P_{j}^{(1)}\right)\left(V_{j}^{(2)}-\lambda_{j}^{(2)}\right) \psi_{j}^{(0)} \\
& +\left(P-P_{j}^{(1)}\right)\left(V_{j}^{(1)}-\lambda_{j}^{(1)}\right)\left(P-P_{j}^{(1)}\right) \psi_{j}^{(1)}
\end{aligned}
$$

Since $P \psi_{j}^{(1)}$ is arbitrary in (17), we are free to make the adjustment

$$
\begin{aligned}
& \left(P-P_{j}^{(1)}\right) \psi_{j}^{(1)}=-S_{j}^{(1)}\left(V_{j}^{(2)}-\lambda_{j}^{(2)}\right) \psi_{j}^{(0)}, \\
& P_{j}^{(1)} \psi_{j}^{(1)} \text { arbitrary } .
\end{aligned}
$$


Then (21) shows that (24) vanishes. Now $C_{j}^{(2)}$ is given by (16), and once again its annihilation can be completed by choosing $\psi_{j}^{(2)}$ as in (17).

It should be observed that the adjustments (23) and (25) to $\psi_{j}^{(0)}$ and $\psi_{j}^{(1)}$ do not make sense without the second-order assumption $\mathscr{C} \subset \mathscr{D}\left(V^{(1)} S V^{(1)}\right)$. However, the components adjusted in (23) and (25) do not affect the first-order coefficients $C_{j}^{(1)}$.

The situation with $p=3,4, \cdots$ is analogous, and the details will be omitted. Briefly, for any such $p$, we would suppose inductively that the condition mentioned in the case $m=1$ (with $p-1$ instead of $p$ ) would permit the choice of $\lambda_{j}^{(1)}, \cdots, \lambda_{j}^{(p-1)}, \psi_{j}^{(0)}, \cdots, \psi_{j}^{(p-1)}$ so that $C_{j}^{(1)}=\cdots=C_{j}^{(p-1)}=0, j=1, \cdots, m$. At this point we would have defined projections $P \geqq P_{j}^{(1)} \geqq \cdots \geqq P_{j}^{(p-1)}$ and operators $V^{(1)}, V_{j}^{(2)}$, $\cdots, V_{j}^{(p-1)}$ with properties analogous to $(20)$. At this point, moreover, the components $P_{j}^{(r)} \psi_{j}^{(p-1-r)}$ would be arbitrary, $r=0,1, \cdots, p-1$, save for the restriction $\left(\psi_{j}^{(0)}, \psi_{k}^{(0)}\right)=\delta_{j k}$. Passing to the $p$ th-order assumption on $\mathscr{D}$, we could then substitute into $B_{j}^{(p)}$ all the previous choices $\psi_{j}^{(0)}, \cdots, \psi_{j}^{(p-1)}$. As in (22), this would lead to the definition of an operator $V_{j}^{(p)}$, a projection $P_{j}^{(p)}$, and a number $\lambda_{j}^{(p)}$, for each $j$, such that an adjustment to $\psi_{j}^{(0)}$ analogous to (23) would kill $P_{j}^{(p-1)} B_{j}^{(p)}$. Next, adjustments like (25) to the components $\left(P_{j}^{(r)}-P_{j}^{(r+1)}\right) \psi_{j}^{(p-1-r)}$ would kill $\left(P_{j}^{(r)}-P_{j}^{(r+1)}\right) B_{j}^{(r+1)}, r=0,1, \cdots, p-2$, leaving (16) in force with $r=p$. Then the choice of $\psi_{j}^{(p)}$ in (17) would kill $C_{j}^{(p)}$, completing the induction, and at this point the components $P_{j}^{(r)} \psi_{j}^{(p-r)}$ would remain arbitrary, $r=0,1, \cdots, p$. Again it should be observed that the adjustments to $\psi_{j}^{(0)}, \cdots, \psi_{j}^{(p-1)}$ made at the $p$ th stage would require the pth-order assumption on $\mathscr{D}$ but would have no effect on $C_{i}^{(1)}, \cdots, C_{j}^{(p-1)}$.

Finally, if this process is carried out to the $p$ th stage, the $\psi_{j}^{(p)}$ can be adjusted as in $\left(12^{\prime}\right)$ so that each $\left(\lambda_{j \kappa}, \varphi_{j \kappa}=\left\|\psi_{j \kappa}^{\prime}\right\|^{-1} \psi_{j \kappa}^{\prime}\right)$ is a $p$ pair for $H_{\kappa}$; and by the orthogonality of the $\psi_{j}^{(0)},\left\{\varphi_{1 \kappa}, \cdots, \varphi_{m k}\right\}$ is an asymptotic basis of order $p$ for $E_{\kappa}[J]$.

To summarize, we may state

LEMmA 3.2. Under Assumption 3.1, the perturbation method yields an asymptotic basis of order $p$ for $E_{\kappa}[J]$, provided that M is contained in the domain of any p-fold product of operators, each of whose factors is either $V$ or $S V$.

In the degenerate case, were it not for the repeated adjustments to components already chosen, much simpler closed expressions could be written down for the $\lambda_{j}^{(r)}$ and $\psi_{j}^{(r)}$. The need for the adjustments to $\psi_{j}^{(r)}$ at stage $r+1$ is evidently due to the appearance of several distinct coefficients $\lambda_{j}^{(1)}$, or equivalently, of several distinct projections $P_{j}^{(1)}<P$, a phenomenon known as splittting. Further splitting, and 
hence further adjustments, might occur at any stage, since the only restriction is that at most $m-1$ of the inequalities $P \geqq P_{j}^{(1)} \geqq P_{j}^{(2)} \geqq \cdots$ can be strict. Thus, although there must in every case be some integer $q$ such that $P_{j}^{(q)}=P_{j}^{(q+1)}=\cdots$, for $j=1, \cdots, m$, it may be difficult in any particular case to say just what $q$ is. Of course $q=1$ if it happens that all the $\lambda_{j}^{(1)}$ are distinct, for then each $P_{j}^{(1)}$ is onedimensional and perforce equal to all the $P_{j}^{(r)}(r>1, j$ fixed); but this instance is by no means typical. In some cases of practical importance, $H$ and $H_{\kappa}$ (and hence all the $V_{j}^{(r)}$ ) are invariant under the action of some linear group $G$ on $\mathscr{C}$. Then the range of each $P_{j}^{(r)}$ is the space of some representation of $G$, and if for some $r=q$ this representation is irreducible, we must have $P_{j}^{(q)}=P_{j}^{(q+1)}=\ldots$ (see [9], pp. 120ff.). The example in $\S 5$ is of this type, where $q$ turns out to be 2 .

Even under Assumption 3.1 it might happen that $\mathscr{C}$ is not contained in $\mathscr{D}(V)$, so that Lemma 3.2 does not apply. In such a case the following observation might be of use.

Lemma 3.3. Under alternative (b) of Assumption 3.1, $E_{\kappa}[J]$ has an asymptotic basis of order $1 / 2$, provided that M It is contained in the domain of $H_{\kappa}^{1 / 2}$ for some $\kappa>0$.

Proof. Under these conditions $\lambda \neq 0$ and $\lambda^{-1}$ is an eigenvalue of $H^{-1}$ with eigenspace $\mathscr{C}$, and it can be proved ([6], pp. 189-191) that, for each unit vector $\psi \in \mathscr{K},\left(\lambda^{-1}, \psi\right)$ is a 1/2-pair for $H_{\kappa}^{-1}$. Denoting the spectral family of $H_{\kappa}^{-1}$ by $\widetilde{E}_{\kappa}$ and applying Lemma 1.3, we get $\left(1-\widetilde{E}_{\kappa}\left[\lambda^{-1}, o\left(\kappa^{1 / 2}\right)\right]\right) \psi=o(1)$, where $o\left(\kappa^{1 / 2}\right)$ is suitably chosen as in Lemma 2.2. Since $E_{\kappa}(\mu)=-\widetilde{E}_{\kappa}(1 / \mu)$ and $\left(\lambda^{-1} \pm o\left(\kappa^{1 / 2}\right)\right)^{-1}=\lambda \mp o\left(\kappa^{1 / 2}\right)$, this means that $\left.\left(1-E_{\kappa} \mid \lambda, o\left(\kappa^{1 / 2}\right)\right]\right) \psi=o(1)$. We put $\psi_{\kappa}=E_{\kappa}\left[\lambda, o\left(\kappa^{1 / 2}\right)\right] \psi$, so that $\psi_{k} \rightarrow \psi$, and it follows from the spectral formula that $\left(H_{\kappa}-\lambda\right) \psi_{\kappa}=o\left(\kappa^{1 / 2}\right)$. Thus $\left(\lambda, \varphi_{\kappa}=\left\|\psi_{\kappa}\right\|^{-1} \psi_{\kappa}\right)$ is a 1/2-pair for $H_{\kappa}$, and it is clear that an asymptotic basis of order $1 / 2$ for $E_{\kappa}[J]$ can be constructed in this way starting with a basis for $\mathscr{l}$.

The hypothesis $\psi \in \mathscr{D}\left(H_{\kappa}^{1 / 2}\right)$ is equivalent with the existence of a sequence $\theta_{n} \in \mathscr{Z}$ such that $\theta_{n} \rightarrow \psi$ and $\left((H+\kappa V)\left(\theta_{n}-\theta_{m}\right), \theta_{n}-\theta_{m}\right)$ is Cauchy; it is weaker than $\psi \in \mathscr{D}(V)$. If $H_{\kappa} \mid \mathscr{D}$ happens to be essentially self-adjoint for some $\kappa>0$, then $\psi \in \mathscr{D}\left(H_{k}^{1 / 2}\right)$ follows from the existence of $V^{1 / 2} \psi([6]$, p. 189。).

4. The case of a stable eigenvalue. Under Assumption 2.3 it may happen that $E_{\kappa}[J]$ is $m$-dimensional for sufficiently small $\kappa$. In this case $\lambda$ is called stable under the perturbation of $H$ to $H_{\kappa}: \Sigma_{\kappa}(J)$ must consist of precisely $m$ eigenvalues $\Lambda_{1 \kappa}, \cdots, A_{m \kappa}$ (counted with their multiplicities) with associated orthonormal eigenvectors $\Phi_{1 \kappa}, \cdots, \Phi_{m \kappa}$. Since $E_{\kappa}[J] \stackrel{s}{\longrightarrow} P$ and $E_{\kappa}[J]$ is $m$-dimensional, it follows that $E_{\kappa}[J] \rightarrow P$ 
in the uniform operator norm. Hence

$$
\left\|(1-P) \Phi_{j \kappa}\right\|=\left\|\left(E_{\kappa}[J]-P\right) \Phi_{j \kappa}\right\| \leqq\left\|E_{\kappa}[J]-P\right\| \rightarrow 0,
$$

and $\left\{\Phi_{1 \kappa}, \cdots, \Phi_{m \kappa}\right\}$ is an asymptotic basis (of any order) for $E_{\kappa}[J]$. Then $\Lambda_{j \kappa} \rightarrow \Lambda$ by Lemma 2.6. More precise estimates follow under the hypotheses of Lemma 3.2 .

Theorem 4.1 Under the hypotheses of Lemma 3.2, let $\left(\lambda_{j \kappa}, \varphi_{{ }_{j \kappa}}\right)$, $j=1, \cdots, m$, be the p-pairs for $H_{\kappa}$ computed by the perturbation method. If $\lambda$ is stable then $\Sigma_{\kappa}(J)$ consists of $m$ eigenvalues $\Lambda_{j \kappa}=$ $\lambda_{j \kappa}+o\left(\kappa^{p}\right)$. Each corresponding eigenvector $\Phi_{j \kappa}$ is within o( $\left.\kappa^{p-q}\right)$ of a linear combination of those $\varphi_{i \kappa}$ for which $\lambda_{i \kappa}$ agrees with $\lambda_{j \kappa}$ up to the $q$ th order (i.e. $\lambda_{i}^{(r)}=\lambda_{j}^{(r)}$ for $r \leqq q$ ).

Proof. The estimate of the $\Lambda_{j \kappa}$ follows from Corollary 2.9.

For each $j=1, \cdots, m$, let $S_{q}(j)$ denote the set of all integers $i$ such that $\lambda_{i \kappa}$ has the same coefficients as $\lambda_{j \kappa}$ up through the order $\kappa^{q}$. If $i \notin S_{q}(j)$ then $\lambda_{i \kappa}$ and $\lambda_{j \kappa}$ have different coefficients of some power $\kappa^{r}$ with $r \leqq q$, and so $\left|\lambda_{i \kappa}-\lambda_{j \kappa}\right|>M \kappa^{q}$ for some $M>O$ and sufficiently small $\kappa$. Then the interval $I_{\kappa}=\left\{\mu:\left|\mu-\lambda_{j \kappa}\right|<M \kappa^{q}\right\}$ contains precisely those $\lambda_{i \kappa}$ and $\Lambda_{i \kappa}$ for which $i \in S_{q}(j)$. Lemma 1.3 gives, for each such $i,\left(1-E_{\kappa}\left[I_{\kappa}\right]\right) \varphi_{i \kappa}=o\left(\kappa^{p-q}\right)$, so that the vectors

$$
\psi_{i \kappa} \equiv E_{\kappa}\left[I_{\kappa}\right] \varphi_{i \kappa}=\varphi_{i \kappa}+o\left(\kappa^{p-q}\right), i \in S_{q}(j),
$$

are eventually linearly independent. But $E_{\kappa}\left[I_{k}\right]$ is the projection on the linear span of the $\Phi_{i \kappa}$ with $i \in S_{q}(j)$, and so the $\psi_{i \kappa}$ also span the range of $E_{\kappa}\left[I_{\kappa}\right]$. Hence, in particular, $\Phi_{j \kappa}$ is a linear combination of the $\psi_{i \kappa}=\varphi_{i \kappa}+o\left(\kappa^{p-q}\right)$ with $i \in S_{q}(j)$, and the theorem is proved.

Since in general all the $\lambda_{i \kappa}$ agree at the zeroth order, each $\Phi_{j \kappa}$ can be written as a linear combination of all the $\varphi_{j \kappa}$ with an error $o\left(\kappa^{p}\right)$. Of course if $m=1$ then $\Phi_{1 \kappa}=\varphi_{1 \kappa}+o\left(\kappa^{p}\right)$. But if $m>1$, even if $\lambda_{j \kappa}$ splits away from all the other $\lambda_{i \kappa}$ at the first order $\left(S_{1}(j)=\{j\}\right)$, the estimate in the theorem is only $\Phi_{j \lambda}=\varphi_{j \lambda}+o\left(\kappa^{p-1}\right)$, and the coefficient of $\kappa^{p}$ in $\varphi_{j \kappa}$ is not given any significance. Better estimates of the $\Phi_{j \kappa}$ evidently require more careful choice of the $\varphi_{j \kappa}$ than suffices merely to make them pseudoeigenvectors of order $p$.

There is a more serious defect in the estimates of the $\Lambda_{j \kappa}$, for $p$ th order assumptions on $\mathscr{C}=E[\lambda] \mathscr{C}$ (see Lemma 3.2) actually guarantee the existence of coefficients $\lambda_{j}^{(r)}$ for $r=1, \cdots, p, \cdots, 2 p$, all of which are significant in estimating perturbed stable eigenvalues ([6], pp. 182-184). Such estimates to within $o\left(\kappa^{2 p}\right)$ cannot be based solely on the theory of pseudoeigenvectors developed here.

The stability of $\lambda$ is a rather delicate matter, and there is no 
abundance of useful sufficient conditions. A well-known one is that $H_{\kappa} \geqq H \geqq c$ as in Lemma $3.1(\mathrm{~b})$, and that the part of the spectrum of $H$ lying to the left of $\lambda$ consist entirely of isolated eigenvalues of finite multiplicity ([6], p. 181.).

5. The Stark effect in the hydrogen atom. We consider as an example the quantum mechanical system of a hydrogen atom in a uniform electric field. Here $\mathscr{\mathscr { C }}=L_{2}\left(R^{3}\right)$. Regarding the electric field as a perturbation, we consider a basic system governed by the Hamiltonian operator of the hydrogen atom in free space. With spin neglected, this is formally

$$
T=-\Delta-c / r,
$$

where $\Delta$ is the three-dimensional Laplacian, $c$ is a positive constant, and $r$ is the distance to the origin. With $\mathscr{D}(T)=C_{0}^{\infty}\left(R^{3}\right), T$ is essentially self-adjoint (see [5]), and we take the unperturbed Hamiltonian $H$ to be the closure $\widetilde{T}$. From the boundedness of $c / r$ relative to $\Delta$ it follows that $\mathscr{D}(H)$ is identical with $\mathscr{D}(\Delta)$ and is contained in $\mathscr{D}(c / r)$, where the last two are the domains of the self-adjoint extensions of $\Delta$ and $c / r$ defined by closure from $C_{0}^{\infty}\left(R^{3}\right)$. Thus it is meaningful to write $H \psi=-\Delta \psi-(c / r) \psi$ for $\psi \in \mathscr{D}(H)$; this will be useful later.

The spectrum of $H$ is well known (e.g. [11] p. 133): the continuous spectrum covers the nonnegative real axis, and the point spectrum consists of countably many isolated negative eigenvalues $\lambda(n)=c^{2} / 4 n^{2}$, $n=1,2, \cdots$, where the multiplicity $m(n)$ of $\lambda(n)$ is $n^{2}$ and each eigenspace $\mathscr{C}(n)$ consists of functions which decay exponentially at infinity ([11], p. 282).

If coordinates are chosen so that the electric field is parallel to the $x_{3}$-axis then the perturbation of the Hamiltonian by the electric field is formally $\kappa x_{3}$, where $\kappa$ is a positive number proportional to the field strength. We define the operator $V$ to be multiplication by $x_{3}$, on the domain

$$
\begin{gathered}
\mathscr{D}(V)=\left\{\varphi \in L_{2}\left(R^{3}\right): \exists R \geqq 0, \exists C \geqq 0, \exists \eta>0 \ni|\varphi(x)| \leqq C e^{-\eta|x|}\right. \\
\text { for almost every } x \text { with }|x| \geqq R\} .
\end{gathered}
$$

Then $C_{0}^{\infty}\left(R^{3}\right) \subset \mathscr{D}(H) \cap \mathscr{D}(V)$, and $H+\kappa V$ defined on $C_{0}^{\infty}\left(R^{3}\right)$ has a self-adjoint extension (in fact a unique one [5]) which we take as the perturbed Hamiltonian $H_{\kappa}$. By Lemma 2.1 (a), $H_{\kappa} \stackrel{r}{\longrightarrow} H$.

For each $\kappa>0, H_{\kappa}$ has no eigenvalues, and the continuous spectrum of $H_{k}$ covers the whole real axis ([11], pp. $134 \mathrm{ff}$.). However, by Corollary 2.10 the spectrum of $H_{\kappa}$ near each $\lambda(n)$ is concentrated to order 0; and we shall now show that this concentration in fact holds to any positive order. 
Clearly Assumption 3.1 (a) holds for each $\lambda=\lambda(n)$; moreover, $\mathscr{L}=\mathscr{C}(n) \subset \mathscr{D}(V)$ since the eigenvectors of $H$ all decay exponentially; and $V \mathscr{D}(V) \subset \mathscr{D}(V)$ by inspection. Thus to verify the hypotheses of Lemma 3.2 for all integers $p$, it suffices to show that $S \mathscr{D}(V) \subset \mathscr{D}(V)$, where $S$ is the reduced resolvent of $H$ at $\lambda$. For as soon as this is known, it is clear that any composite of finitely many factors $S$ and $S V$ can be applied to each $\varphi \in \mathscr{D}(V)$.

To see that $S \mathscr{D}(V) \subset \mathscr{D}(V)$, fix $\varphi \in \mathscr{D}(V)$. Since $P \varphi \in \mathscr{C} \subset \mathscr{D}(V)$, it is enough to consider the case in which $\varphi$ is orthogonal to $\mathscr{K}$, i.e. $(1-P) \varphi=\varphi$. Now $\psi=S \varphi \in \mathscr{D}(H)$, and

$$
(H-\lambda) \psi=(H-\lambda) S \varphi=(1-P) \varphi=\varphi .
$$

According to the remark after the definition of $H$, this equality can be written

$$
(-\Delta-\lambda) \psi=\varphi+(c / r) \psi .
$$

The operator on the left has a bounded inverse, given by convolution with the function $(4 \pi r)^{-1} e^{-k r}$, where $-\lambda=2 k^{2}, k>0$. Applying the inverse to the above relation, we obtain

$$
\psi(x)=\frac{1}{4 \pi} \int_{R^{3}} \frac{e^{-k|x-y|}}{|x-y|}\left[\varphi(y)+\frac{c}{|y|} \psi(y)\right] d y
$$

for almost all $x \in R^{3}$. Since our aim is to show that $\psi$ decays exponentially at infinity, it suffices to estimate $\psi$ in the exterior $B^{\prime}$ of some ball $B=B(R)$ with center at the origin and radius $R$. To this end we write (26) as

$$
\psi(x)=\xi(x)+\frac{1}{4 \pi} \int_{B^{\prime}} \frac{e^{-k|x-y|}}{|x-y|} \frac{c}{|y|} \psi(y) d y,
$$

where $\xi(x)$ is the sum of the contributions of $\psi$ and $\phi$ to the integral in (26) over $B$ and the contribution of $\varphi$ to the integral over $B^{\prime}$. Treating each of these contributions separately, taking $R$ sufficiently large, and using the fact that $\psi \in \mathscr{C}$ and $\psi \in \mathscr{D}(V)$, together with Schwarz' inequality and various elementary inequalities on $|x-y|$, one can show that $\xi \in \mathscr{D}(V)$, i.e.

$$
|\xi(x)| \leqq A(R) e^{-\gamma|x|}
$$

for $|x| \geqq R$, where $0<\gamma<(1 / 2) k$, and $A(R)$ is a constant which grows with $R$. It should be noted that $\psi$ is already a well-defined item, namely $S \varphi$, and that (27) expresses one of its properties; there is no question of solving (27) for an unknown $\psi$.

Now we regard the right side of (27) as defining a map $K$ in 
$L_{2}\left(B^{\prime}\right)$. A straightforward estimate shows that this is true, and in fact that $K$ is a strict contraction map if $R$ is sufficiently large. Thus $K$ has a unique fixed point given by $\bar{\psi}=\lim K^{n} \Phi$, where $\Phi$ is any element whatever in $L_{2}\left(B^{\prime}\right)$. With $\Phi(x)=0, K \Phi=\xi$ obeys (28), and it can be shown easily by induction that, for $R$ sufficiently large, $K^{n} \Phi$ is dominated by $2 A(R) e^{-\gamma|x|}$. Hence so is the fixed point $\bar{\psi}$.

But (27) asserts in particular that $\psi$ restricted to $B^{\prime}$ is a fixed point of $K$, and as such it must agree almost everywhere with $\bar{\psi}$. Hence $\psi$ decays exponentially, and we have shown that $\psi=S \varphi \in \mathscr{D}(V)$ if $\varphi \in \mathscr{D}(V)$.

It follows from Lemma 3.2 that, for each eigenvalue $\lambda(n)$ of $H$ and each positive integer $p$, the perturbation method yields an asymptotic basis of order $p$ for $E_{n}[J(n)]$, where $J(n)$ is an isolating interval for $\lambda(n)$. Theorem 2.7 then shows that $\Sigma_{\kappa}(J(n))$ is concentrated on the union $C_{\kappa}(n)$ of intervals of width $o\left(\kappa^{p}\right)$ centered on the pseudoeigenvalues $\lambda_{j_{k}}(n)$ computed by the perturbation method.

In passing, we remark that these pseudoeigenvalues have been calculated at least up to the second order (e.g. [8], p. 270). At the first order, $\lambda(n)$ splits into $2 n-1$ distinct pseudoeigenvalues, the different $\lambda_{i}^{(1)}$ being proportional to $n i,-n+1 \leqq i \leqq n-1$, and having multiplicities (as eigenvalues of $P(n) V P(n)$ ) equal to $n-|i|$. At the second order, further splitting occurs: if $n-|i|$ is even, $\lambda_{i}^{(1)}$ corresponds to $(1 / 2)(n-|i|)$ distinct $\lambda_{j}^{(2)}$ all of multiplicity 2 , and if $n-|i|$ is odd, $\lambda_{i}^{(1)}$ corresponds to $(1 / 2)(n-|i|+1)$ distinct $\lambda_{j}^{(2)}$, of which one has multiplicity 1 and the rest have multiplicity 2. Altogether, at the second order, $\lambda(n)$ has split into $n$ "simple" pseudoeigenvalues and $(1 / 2) n(n-1)$ "double" ones. Now, the symmetry group of $H_{\kappa}$ is generated by all rotations of $R^{3}$ about the $x_{3}$-axis together with reflections in a plane containing the $x_{3}$-axis, and its irreducible representations are all one-and two-dimensional. The $n^{2}$-dimensional representation of this group in the eigenspace $\mathscr{C}(n)$ contains $n$ copies of the first kind of irreducible representation, and $(1 / 2) n(n-1)$ copies of the second kind (see, e.g. [22], pp. 204-205). These correspond exactly to the multiplicities of the $\lambda_{j}^{(2)}$ and so $\lambda(n)$ cannot split further past the second order.

Returning to the concentration of the spectrum of $H_{n}$, we can write the negative real axis $N=(-\infty, 0)$ as the union of closed intervals $J(n)$, each of which isolates $\lambda(n), n=1,2, \cdots$. By considering the dense family $\mathfrak{F}$ of finite linear combinations of vectors in $\bigcup_{n=1}^{\infty} \mathscr{C}(n)$ and using the uniform boundedness of the projections $E_{\kappa}\left[\left(C_{\kappa}(n)\right]\right.$, we easily see that $E_{\kappa}\left[N-C_{\kappa}\right] \stackrel{s}{\longrightarrow} 0$, where $C_{\kappa}=\bigcup_{n=1}^{\infty} C_{\kappa}(n)$. In other words, for any positive integer $p$, the negative spectrum of $H_{\kappa}$ is concentrated on the union of intervals of length o( $\left.\kappa^{p}\right)$ centered 
on the numbers $\lambda_{j k}(n)$ computed by applying the perturbation method to the unperturbed eigenvalues $\lambda(n)$ (cf. [2], p. 20).

This property distinguishes the $\lambda_{j k}(n)$ from other negative points of the spectrum of $H_{k}$. It is frequently pointed out that, although the system governed by $H_{k}$ has no stationary states in the strict sense (since $H_{k}$ has no eigenvalues), nonetheless the $\lambda_{j k}$ represent the energies of "almost stationary" states (see, e.g. [8], p. 274 fn.; [12], p. 206). Indeed, if $\left(\lambda_{\kappa}, \varphi_{\kappa}\right)$ is any $p$-pair for $H_{k}$, then by estimating the spectral formula in two parts, off and on the interval $\lambda_{\kappa}-\kappa^{p} \leqq \mu \leqq \lambda_{\kappa}+\kappa^{p}$, we obtain

$$
\left\|e^{-i t H_{\kappa}} \varphi_{\kappa}-e^{-i t \lambda_{\kappa}} \varphi_{\kappa}\right\|^{2} \leqq 4\left\|\left(1-E_{\kappa}\left[\lambda_{\kappa}, \kappa^{p}\right]\right) \varphi_{\kappa}\right\|^{2}+\left(t \kappa^{p}\right)^{2} .
$$

If $\varepsilon>0$ is specified, Lemma 1.3 shows that $\kappa_{0}>0$ can be chosen so that the first term on the right is $<\varepsilon^{2} / 2$ for $\kappa<\kappa_{0}$. Then the second term remains $<\varepsilon^{2} / 2$ for all $t \leqq T=(\varepsilon / \sqrt{2}) h^{-p}$. In other words, for $\kappa$ small enough, the state $e^{-i t H_{\kappa}} \varphi_{\kappa}$ remains with in $\varepsilon$ in norm of its original value $\varphi_{\kappa}$ for all times in an interval $[0, T]$ whose length increases with $1 / \kappa^{p}$.

It is stressed that this result holds for an arbitrary $p$-pair of $H_{\kappa}$; and since $H_{\kappa}$ has continuous spectrum everywhere, a $p$-pair $\left(\lambda_{\kappa}, \varphi_{\kappa}\right)$ can be constructed in which $\lambda_{\kappa}$ has any value whatever. Thus the "almost stationary" property does not by itself characterize the pairs $\left(\lambda_{j k}(n), \varphi_{j \kappa}(n)\right)$.

However, in view of the concentration of $\Sigma_{k}(N)$ near the $\lambda_{j k}(n)$, we see by Lemma 1.4 that if $\left(\lambda_{\kappa}, \varphi_{\kappa}\right)$ is a $p$-pair whose $\varphi_{\kappa}$ has no sequence $\varphi_{\kappa_{n}} \stackrel{w}{\longrightarrow} 0\left(\kappa_{n} \rightarrow 0\right)$, then $\lambda_{\kappa}$ must be within $o\left(\kappa^{p}\right)$ of the set of all $\lambda_{j k}(n)$. We may conclude that the $\lambda_{j \kappa}(n)$ represent the only possible energies which are continuous in $\kappa$ and whose corresponding states $\varphi_{j \kappa}(n)$ are both "almost stationary" and remain outside some weak neighborhood of 0 . The last condition rules out wave functions which, though technically "almost stationary", have supports which shrink to a point or migrate to infinity as $\kappa$ is reduced.

\section{REFERENCES}

1. C. C. Conley, and P. A. Rejto, On Spectral Concentration, Cour. Inst. Math. Sci., New York Univ., Research Report IMM-293, 1962.

2. - A Note on Spectral Concentration Phenomena, Math. Res. Center, Madison. Wisconsin, Report No. 602 AD, 1965.

3. K. O. Friedrichs, On the perturbation of continuous spectra, Comm. Pure Appl. Math. 1 (1948), 361-406.

4. K. O. Friedrichs, and P. A. Rejto, On a perturbation through which a discrete spectrum becomes continuous, Comm. Pure Appl. Math. 15 (1962), 219-235.

5. T. Ikebe, and T. Kato, Uniqueness of the self-adjoint extension of singular elliptic differential operators, Arch. Rat. Mech. Anal. 9 (1962), 77-92. 
6. T. Kato, On the convergence of the perturbation method, J. Fac. Sci. Univ. Tokyo Sect. I, 6 (1951), 145-226.

7. - Perturbation Theory for Linear Operators, Springer-Verlag, 1966.

8. L. D. Landau, and E. M. Lifshitz, Quantum Mechanics: non-relativistic Theory, Trans. J. B. Sykes and J. S. Bell, 2nd rev. ed., Pergammon Press, 1965.

9. G. W. Mackey, Mathematical Foundations of Quantum Mechanics, Math. Physics Monograph Series, W. A. Benjamin, 1963.

10. E. C. Titchmarsh, Some theorems on perturbation, J. Analyse Math. 4, (1954), 187-208.

11. —, Eigenfunction Expansions Associated with Second-order Differential Equations, Part II, Oxford Univ. Press 1958.

12. E. P. Wigner, Group Theory and its Application to the Quantum Mechanics of Atomic Spectra, Trans. J. J. Griffin, Academic Press, 1959.

Received April 30, 1966. Part of this work was supported by National Science Foundation Grant G-22982 and Air Force of Scientific Research Grant 553-64. 



\section{PACIFIC JOURNAL OF MATHEMATICS}

\section{EDITORS}

\section{H. ROYDEN \\ Stanford University \\ Stanford, California}

\author{
J. P. JANS \\ University of Washington \\ Seattle, Washington 98105
}

J. DugundJI

Department of Mathematics

Rice University

Houston, Texas 77001

RichaRd ARENS

University of California

Los Angeles, California 90024

\section{ASSOCIATE EDITORS}
E. F. BECKENBACH
B. H. NeumanN
F. WOLF
K. YOSIDA

\section{SUPPORTING INSTITUTIONS}

\author{
UNIVERSITY OF BRITISH COLUMBIA \\ CALIFORNIA INSTITUTE OF TECHNOLOGY \\ UNIVERSITY OF CALIFORNIA \\ MONTANA STATE UNIVERSITY \\ UNIVERSITY OF NEVADA \\ NEW MEXICO STATE UNIVERSITY \\ OREGON STATE UNIVERSITY \\ UNIVERSITY OF OREGON \\ OSAKA UNIVERSITY \\ UNIVERSITY OF SOUTHERN CALIFORNIA
}

\author{
STANFORD UNIVERSITY \\ UNIVERSITY OF TOKYO \\ UNIVERSITY OF UTAH \\ WASHINGTON STATE UNIVERSITY \\ UNIVERSITY OF WASHINGTON

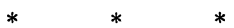 \\ AMERICAN MATHEMATICAL SOCIETY \\ CHEVRON RESEARCH CORPORATION \\ TRW SYSTEMS \\ NAVAL ORDNANCE TEST STATION
}

Mathematical papers intended for publication in the Pacific Journal of Mathematics should be typewritten (double spaced). The first paragraph or two must be capable of being used separately as a synopsis of the entire paper. It should not contain references to the bibliography. Manuscripts may be sent to any one of the four editors. All other communications to the editors should be addressed to the managing editor, Richard Arens at the University of California, Los Angeles, California 90024.

50 reprints per author of each article are furnished free of charge; additional copies may be obtained at cost in multiples of 50 .

The Pacific Journal of Mathematics is published monthly. Effective with Volume 16 the price per volume (3 numbers) is $\$ 8.00$; single issues, $\$ 3.00$. Special price for current issues to individual faculty members of supporting institutions and to individual members of the American Mathematical Society: $\$ 4.00$ per volume; single issues $\$ 1.50$. Back numbers are available.

Subscriptions, orders for back numbers, and changes of address should be sent to Pacific Journal of Mathematics, 103 Highland Boulevard, Berkeley 8, California.

Printed at Kokusai Bunken Insatsusha (International Academic Printing Co., Ltd.), 7-17, Fujimi 2-chome, Chiyoda-ku, Tokyo, Japan.

PUBLISHED BY PACIFIC JOURNAL OF MATHEMATICS, A NON-PROFIT CORPORATION

The Supporting Institutions listed above contribute to the cost of publication of this Journal, but they are not owners or publishers and have no responsibility for its content or policies. 


\section{Pacific Journal of Mathematics}

\section{Vol. 23, No. 2 \\ April, 1967}

Herbert Stanley Bear, Jr. and Bertram John Walsh, Integral kernel for

one-part function spaces .......................... 209

Mario Borelli, Some results on ampleness and divisorial schemes ....... 217

John A. Erdos, Unitary invariants for nests . ................... 229

Nathaniel Grossman, The volume of a totally-geodesic hypersurface in a pinched manifold.................................. 257

D. M. Hyman, A generalization of the Borsuk-Whitehead-Hanner theorem ............................................. 263

I. Martin (Irving) Isaacs, Finite groups with small character degrees and large prime divisors ............................. 273

I. Martin (Irving) Isaacs, Two solvability theorems ................ 281

William Lee Johnson, The characteristic function of a harmonic function in a locally Euclidean space ............................... 291

Ralph David Kopperman, Application of infinitary languages to metric spaces ............................................. 299

John Lauchlin MacDonald, Relative functor representability ............ 311

Mahendra Ganpatrao Nadkarni, A class of measures on the Bohr group.... 321

Keith Lowell Phillips, Hilbert transforms for the p-adic and p-series fields....................................... 329

Norman R. Reilly and Herman Edward Scheiblich, Congruences on regular semigroups ...

Neil William Rickert, Measures whose range is a ball ... . .

Gideon Schwarz, Variations on vector measures

Ronald Cameron Riddell, Spectral concentration for self-adjoint operators. .

Haskell Paul Rosenthal, A characterization of restrictions of

Fourier-Stieltjes transforms ................... 